\title{
Reconstruction and analysis of circRNA-miRNA-mRNA network in the pathology of cervical cancer
}

\author{
YUEXIONG YI, YANYAN LIU, WANRONG WU, KEJIA WU and WEI ZHANG \\ Department of Obstetrics and Gynecology, Zhongnan Hospital of Wuhan University, \\ Wuhan, Hubei 430071, P.R. China
}

Received August 9, 2018; Accepted February 15, 2019

DOI: $10.3892 /$ or.2019.7028

\begin{abstract}
The present study was performed with the aim of understanding the mechanisms of pathogenesis and providing novel biomarkers for cervical cancer by constructing a regulatory circular (circ)RNA-micro (mi)RNA-mRNA network. Using an adjusted P-value of $<0.05$ and an absolute log value of fold-change $>1,16$ and 156 miRNAs from GSE30656 and The Cancer Genome Atlas (TCGA), 5,321 mRNAs from GSE63514, 4,076 mRNAs from cervical squamous cell carcinoma and endocervical adenocarcinoma (from TCGA) and 75 circRNAs from GSE102686 were obtained. Using RNAhybrid, Venn and UpSetR plot, 12 circRNA-miRNA pairs and 266 miRNA-mRNA pairs were obtained. Once these pairs were combined, a circRNA-miRNA-mRNA network with 11 circRNA nodes, 4 miRNA nodes, 153 mRNA nodes and 203 edges was constructed. By constructing the protein-protein interaction network using Molecular Complex Detection scores $>5$ and $>5$ nodes, 7 hubgenes (RRM2, CEP55, CHEK1, KIF23, RACGAP1, ATAD2 and KIF11) were identified. By mapping the 7 hubgenes into the preliminary circRNA-miRNA-mRNA network, a circRNA-miRNA-hubgenes network consisting of 5 circRNAs (hsa_circRNA_000596, hsa_circRNA_104315, hsa_circRNA_400068, hsa_circRNA_101958 and hsa_circRNA_103519), 2 mRNAs (hsa-miR-15b and hsa-miR-106b) and 7 mRNAs (RRM2, CEP55, CHEK1, KIF23, RACGAP1, ATAD2 and KIF11) was constructed. There were 22 circRNA-miRNA-mRNA regulatory axes identified in the subnetwork. By analyzing the overall survival for the 7 hubgenes using the Gene Expression Profiling Interactive Analysis tool, higher expression of RRM2 was demonstrated to be associated with a significantly poorer overall survival. PharmGkb analysis identified single nucleotide polymorphisms
\end{abstract}

Correspondence to: Professor Wei Zhang, Department of Obstetrics and Gynecology, Zhongnan Hospital of Wuhan University, 169 Donghu Road, Wuhan, Hubei 430071, P.R. China E-mail: zw6676@163.com

Key words: non-coding RNA, cervical cancer, bioinformatics, carcinoma, disease makers
(SNPs) of rs5030743 and rs1130609 of RRM2, which can be treated with cladribine and cytarabine. RRM2 was also indicated to be involved in the gemcitabine pathway. The 5 circRNAs (hsa_circRNA_000596, hsa_circRNA_104315, hsa_circRNA_400068, hsa_circRNA_101958 and hsa_ circRNA_103519) may function as competing endogenous RNAs and serve critical roles in cervical cancer. In addition, cytarabine may produce similar effects to gemcitabine and may be an optional chemotherapeutic drug for treating cervical cancer by targeting rs5030743 and rs1130609 or other similar SNPs. However, the specific mechanism of action should be confirmed by further study.

\section{Introduction}

Cervical cancer is the fourth most common cause of fatality that threatens women's health excessively in developing counties (1). Cervical cancer cases were associated with 265,700 fatalities in 2012 (2). Approximately half a million new cases occur worldwide each year (2). Although $80 \%$ of early-stage cervical cancer cases can be treated with surgery, radiotherapy or chemotherapy, there is still a large number of advanced-stage patients with poor prognoses (3). The association between human papillomaviruses (HPV) and cervical cancer has been strongly verified (4); however, the specific mechanisms remain to be fully elucidated. Therefore, further studies on the underlying mechanism of tumor initiation and development are necessary.

Non-coding (nc)RNA is a functional RNA molecule that is transcribed from DNA but not translated into proteins (5). Micro(mi)RNAs are a vital component of the endogenous ncRNA family, which are $\sim 19-25$ nucleotides in length. The majority of miRNAs are highly conserved in sequence and are involved in multiple cellular functions via the post-transcriptional regulation of gene transcription (6). According to previous studies, several miRNAs (7-11) have been consistently reported to be involved in the development of cervical cancer, which suggests that miRNAs are highly correlated with the pathogenesis of cervical cancer.

Competing endogenous RNAs (ceRNAs) are transcripts with the same miRNA response element (MRE) that act as miRNA sequestering molecules and compete to bind to miRNAs to regulate their target genes, thereby affecting the biological behavior of tumors (12). Circular (circ)RNAs are a 
novel class of the ceRNA and a distinctive type of ncRNA that were identified in plant viruses in 1976 (13). Unlike traditional linear RNA, circRNAs are structured as a distinct closed loop. Therefore, circRNAs are not easily degraded and stably expressed in various organisms (14). Following the binding to miRNA by MREs, circRNAs can also serve an important role in the occurrence and development of tumors as tumor suppressors or proto-oncogenes (15). Recently, various studies have indicated that circRNAs are involved in the initiation and development of multiple diseases, including gastric cancer (16), osteoarthritis (17) and hepatocellular carcinoma (18).

As the regulatory gene for miRNAs, more studies have suggested that circRNAs may serve key roles in the carcinogenesis of multiple types of cancer $(16,18,19)$. However, research regarding cervical cancer is limited. Wang et al (20) revealed that hsa-circ-0101996 combined with hsa-circ-0101119 in peripheral whole blood was identified as the potential biomarkers for human cervical squamous cell cancer. Gao et al (21) reported that hsa-circ-0018289 was upregulated in cervical cancer and promotes proliferation, migration and invasion of tumor cells. Furthermore, Ma et al (22) indicated that activated has-circ-000284 promotes cell proliferation and invasion in cervical cancer. This evidence supports that circRNA is likely to participate in the development of cervical cancer, and probably by indirectly regulating the expression of target genes through affecting miRNAs.

The molecular mechanism underlying the role of ncRNAs in the carcinogenesis and progression of cervical cancer remains unknown. Therefore, in order to further understand the potential role of ncRNAs in cervical cancer, the differentially expressed RNAs (including circRNA, miRNA and mRNA) were identified by microarray and databases and a regulatory circRNA-miRNA-mRNA network was constructed (Fig. 1). The present study may illuminate the underlying mechanisms of cervical cancer pathogenesis and provide novel biomarkers and targets for cervical cancer.

\section{Materials and methods}

Raw data. Gene Expression Omnibus (GEO; https://www. ncbi.nlm.nih.gov/geo/) is an international public repository for high-throughput microarray and sequence-based data (23). GSE102686 circRNA microarray, GSE30656 miRNA profiles and GSE63514 mRNA datasets for cervical cancer were downloaded from GEO, respectively. The fundamental information for these three profiles is summarized in Table I. In addition, the miRNA and mRNA expression datasets for cervical squamous cell carcinoma and endocervical adenocarcinoma (CESC), which contained 309 cervical cancer samples with 3 normal samples, were also downloaded from The Cancer Genome Atlas (TCGA) by cBioPortal (http://www.cbioportal.org/) (24).

Identification of differentially expressed (DE)-miRNAs, $D E$-circRNAs and DE-mRNAs. The expression difference between the normal and cervical cancer groups was utilized to determine the DE-circRNAs DE-miRNAs and DE-mRNAs. The adjusted P-value (adj.p) and the absolute log value of fold-change $(\log \mid \mathrm{FCl})$ were calculated using $\mathrm{R}$ software with limma package (25). The criteria of adj.p $<0.05$ and $\log \mid \mathrm{FCl}>1$ were adopted to select the DE-circRNAs, DE-miRNAs and DE-mRNAs.

Construction of miRNA-mRNA pairs. Targeted mRNAs of the collected miRNAs were predicted using miRWalk (Version 2.0; http://zmf.umm.uni-heidelberg.de/apps/zmf/mirwalk2/) (26), which included 12 databases (Microt4, miRWalk, mir-bridge, miRanda, miRDB, miRMap, Pictar2, PITA, miRNAMap, RNAhybrid, RNA22, and TargetScan). To increase the accuracy of prediction, the targeted genes were select using the criteria as follows: i) The targeted genes should be predicted by TargetScan (27) and miRanda (28); and ii) the targeted genes should be overlapped in at least $8 / 12$ databases. The selected targeted mRNAs were merged with DE-mRNAs of CESC and GSE63514. The overlapped gene sets were analyzed with UpSetR (29) and Venn Plot, and the pairs of miRNAs and mRNAs were subsequently constructed.

Construction of circRNA-miRNA pairs. To further predict the target circRNAs, the sequences of the candidate DE-miRNAs and DE-circRNAs were downloaded from miRbase (30) and GSE102686, respectively. Following this, the miRNA targets of circRNAs were predicted, and the minimum free energy of circRNA-miRNA duplexes was calculated using the RNAhybrid program (https://bibiserv2.cebitec.uni-bielefeld. de/rnahybrid) (31). miRNA target binding sites on the whole circRNA sequences were predicted. To obtain high-quality circRNAs acting as miRNA targets and distinguish those circRNAs acting as miRNA decoys, the circRNAs that had perfect nucleotide pairing between the 2 nd and 8 th positions of the 5' end of miRNA sequences were selected (32) and the circRNA-miRNA pairs were identified simultaneously.

Reconstruction of the circRNA-miRNA-mRNA network. The preliminary circRNA-miRNA-mRNA network was reconstructed by combining the pairs of miRNA-mRNA and miRNA-circRNA. The nodes that could not form a circRNA-miRNA-mRNA axis were removed. The circRNA-miRNA-mRNA network was visualized using Cytoscape software (version 3.6.1) (33).

Gene Ontology (GO) and pathway enrichment analysis. GO and pathway enrichment analyses were performed using The Database for Annotation, Visualization and Integrated Discovery (https://david.ncifcrf.gov/) (34) on the differentially expressed genes (DEGs) in the preliminary circRNA-miRNA-mRNA network. The significantly enriched biological items for biological process (BP), cellular components (CC), and molecular functions (MF) were identified with $\mathrm{P}<0.05$. Pathways with $\mathrm{P}<0.05$ were considered as the significantly enriched pathways. The significant GO items and pathways were visualized using Goplot package (35).

Protein-protein interaction (PPI) network analysis. The Search Tool for the Retrieval of Interacting Genes/Proteins (STRING) database (https://string-db.org/) (36) was used to predict the association between target genes in regulatory network analysis. To obtain more accurate results, nodes with interaction score $<0.7$ and that were not connected to the major network were removed. The Molecular Complex Detection 
Table I. Basic information of the three microarray datasets from Gene Expression Omnibus.

\begin{tabular}{lllllclr}
\hline Profile & RNA type & Platform & Organism & Experiment type & $\begin{array}{c}\text { Sample size } \\
\text { (T/N) }\end{array}$ & Region & Year \\
\hline GSE102686 & circRNA & GPL19978 & Homo sapiens & Non-coding RNA profiling by array & $5 / 5$ & China & 2017 \\
GSE30656 & miRNA & GPL6955 & Homo sapiens & Non-coding RNA profiling by array & $19 / 10$ & Netherlands & 2012 \\
GSE63514 & mRNA & GPL570 & Homo sapiens & Expression profiling by array & $28 / 24$ & USA & 2015
\end{tabular}

T, tumor; N, normal.

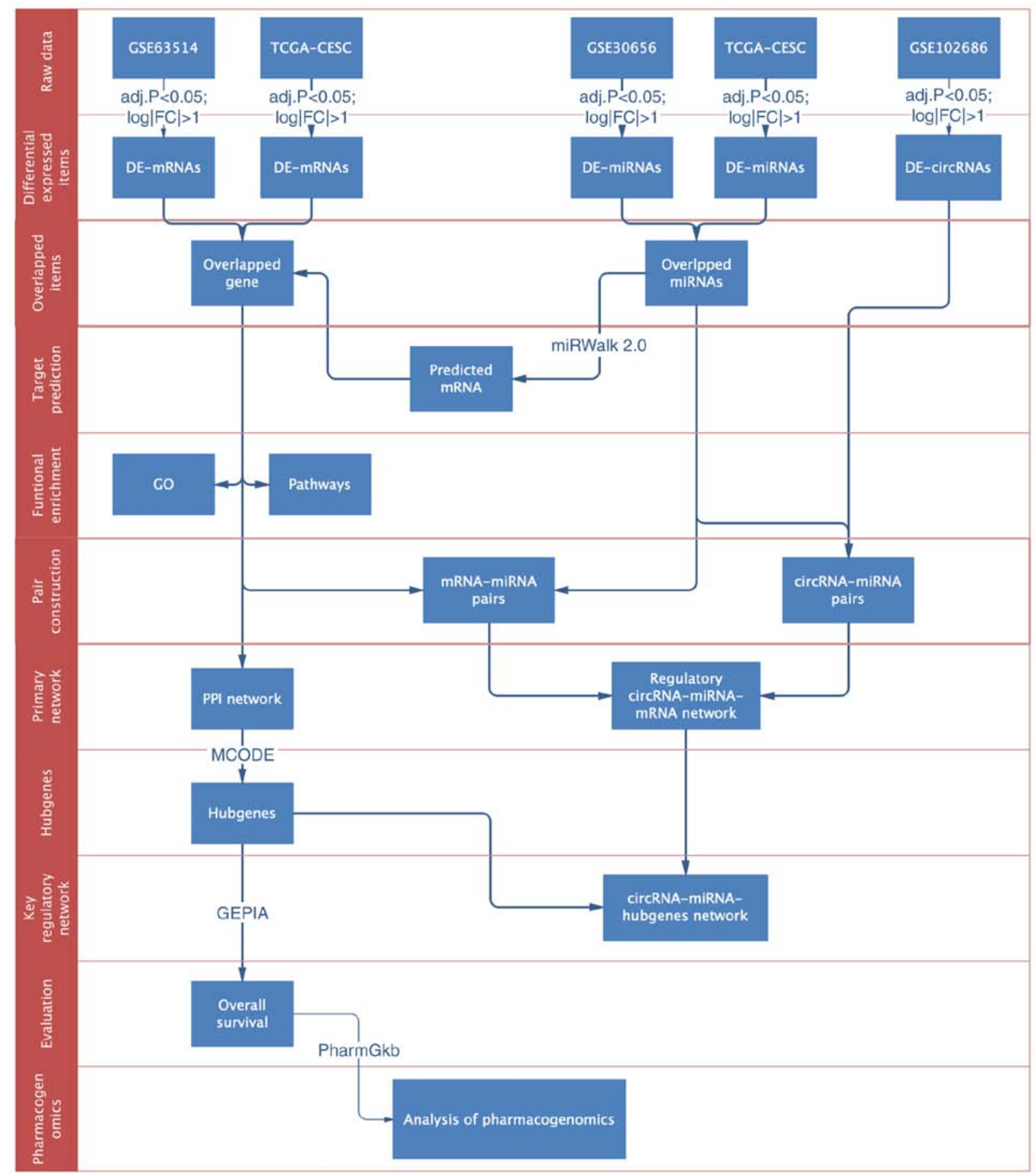

Figure 1. Flowchart of the construction and clinical evaluation of circRNA-miRNA-mRNA network. TCGA, The Cancer Genome Atlas; CESC, cervical squamous cell carcinoma and endocervical adenocarcinoma; adj.p, adjusted P-value; FC, fold-change; DE, differentially expressed; GO, Gene Ontology; PPI, protein-protein interaction network; GEPIA, the database of Gene Expression Profiling Interactive Analysis; circRNA, circular RNA; miRNA, microRNA. 
A

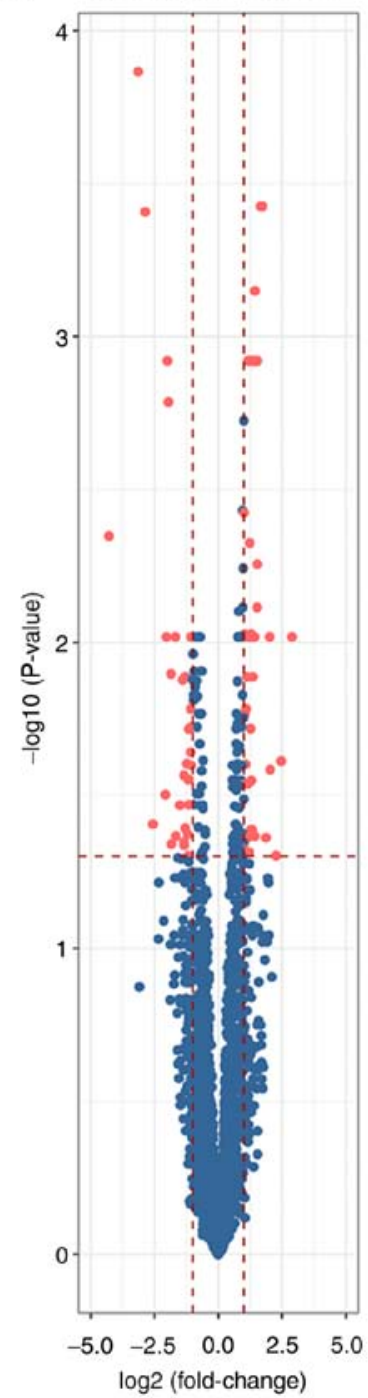

B

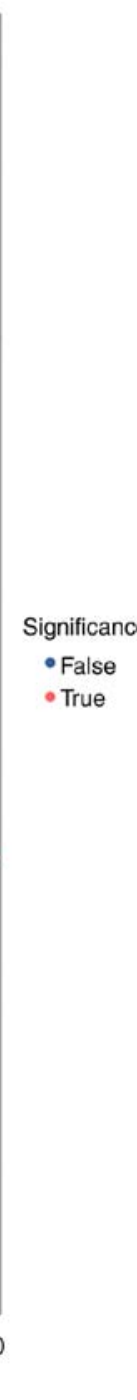

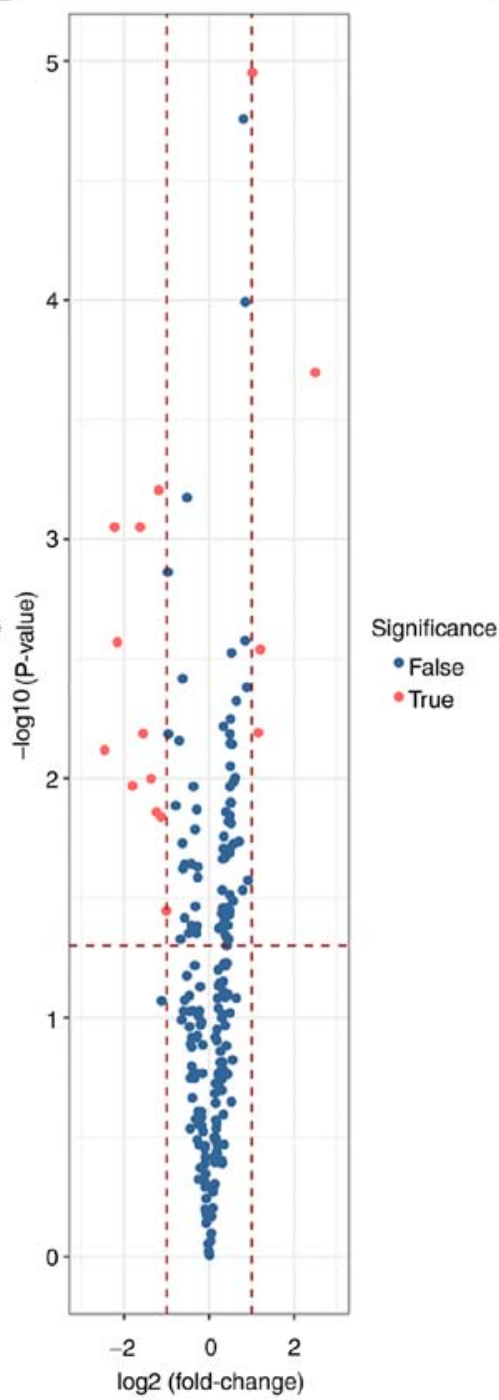

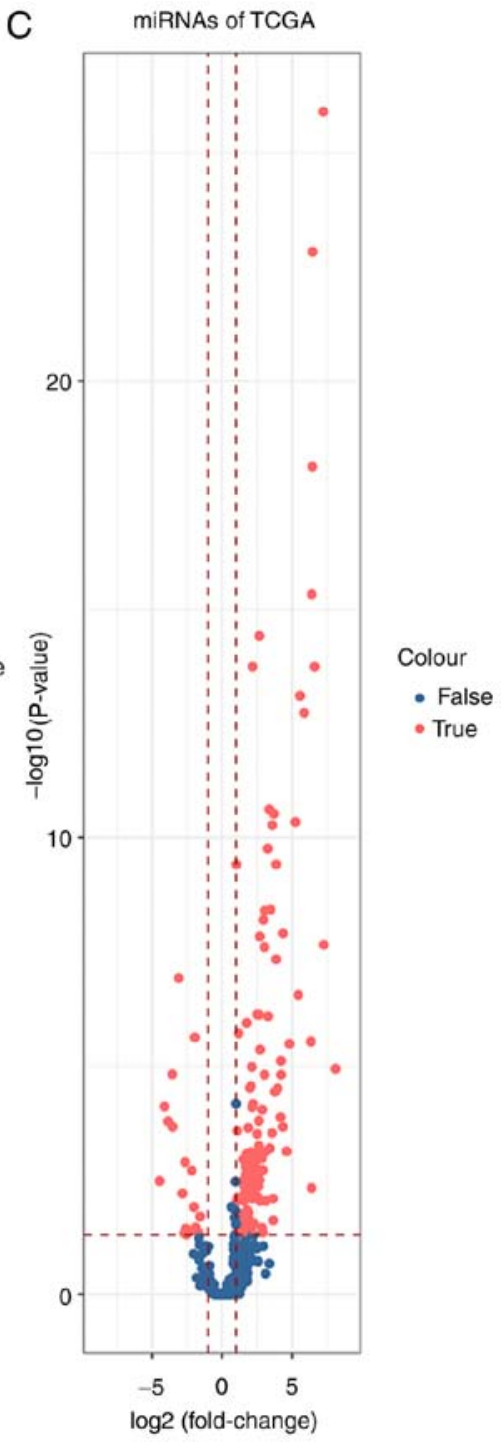

Figure 2. Volcano plots for DE-circRNAs, DE-miRNAs and DE-mRNAs identified from GEO and TCGA. (A) DE-circRNAs from GSE102686; (B) DE-miRNAs from GSE306565; (C) DE-miRNAs from TCGA.

(MCODE) (37) plug-in in Cytoscape was used to analyze PPI network modules, and MCODE scores $>5$ with $>5$ nodes were set as cut-off criteria with the default parameters (Degree cut-off $\geq 2$, node score cut-off $\geq 0.2, \mathrm{~K}$-core $\geq 2$ and max depth $=100$ ). The genes in the cluster were considered hubgenes.

Reconstruction of the circRNA-miRNA-hubgene network. In order to identify the association between DE-circRNAs, DE-miRNAs and hubgenes, the aforementioned hubgenes were mapped into the preliminary circRNA-miRNA-mRNA network and the relevant DE-circRNAs and DE-miRNAs were also extracted. Subsequently, the subnetwork that was identified as the circRNA-miRNA-hubgene network was identified. The structure of circRNAs and the secondary stem-loop structure of miRNAs were also predicted by the cancer-specific circRNA database (CSCD) (38) and Vienna RNA (39), respectively.

Evaluation of overall survival (OS) for hubgenes. To identify the effect of the hubgenes on survival, the Gene Expression Profiling Interactive Analysis (GEPIA) database (40) was utilized to explore the association between these hubgenes and OS. The genes with $\mathrm{P}<0.05$ were considered as critical genes.

Pharmacogenomics analysis for critical genes. Critical genes were analyzed using the database of PharmGkb for the potential single nucleotide polymorphisms (SNPs) and applicable medications, as well as the pharmacogenomics pathway. Specifically, information on clinical and SNP annotations for the critical genes were extracted, including genes names, SNPs, associated medications, efficiency, significance, P-values, genotype association with medications, references and pathways.

\section{Results}

Identification of DE-circRNAs, DE-miRNAs and DE-mRNAs. With the criteria of adj.p $<0.05$ and $\log \mid \mathrm{FCl}>1$ a DE-circRNA dataset consisting of 75 DE-circRNAs, with 43 upregulated and 32 downregulated circRNAs, was identified from GSE102686 (Fig. 2A). A DE-miRNAs dataset, which consisted of 16 and 156 DE-miRNAs, was extracted from GSE30656 and 

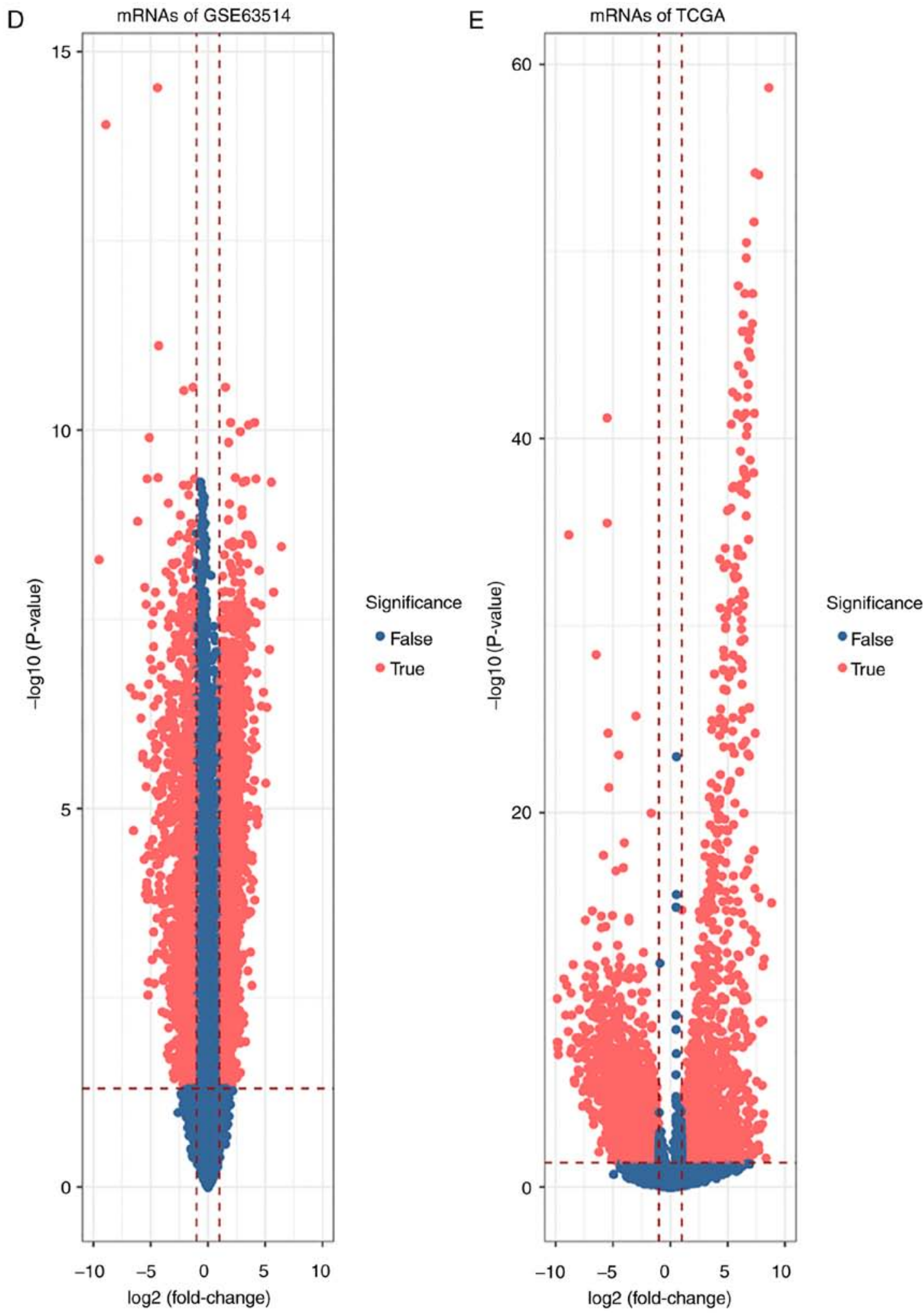

Figure 2. Continued. (D) DE-mRNAs from GSE63514; and (E) DE-mRNA from TCGA. DE, differentially expressed; circRNA, circular RNA; miRNA, microRNA; TCGA, The Cancer Genome Atlas; GEO, Gene Expression Omnibus.

TCGA. By merging these two screening results, 5 miRNAs (hsa-miR-21, hsa-miR-99a, hsa-miR-106b, hsa-miR-15b and hsa-miR-203) were obtained. hsa-miR-106b, hsa-miR-15b and hsa-miR-21 were upregulated whereas hsa-miR-99a was downregulated in CESC and GSE30656. However, hsa-miR-203 was upregulated in CESC but downregulated in GSE30656 (Fig. 2B and C). A DE-mRNA dataset, which consisted of 5,321 and 4,076 DE-mRNAs from GSE63514 and CESC, was determined (Fig. 2D and E). In addition, 3,400 targeted genes predicted by miRWalk with 12 databases were identified. The expression of the 75 DE-circRNAs is indicated in Fig. 3A, and the expression of 5 miRNAs in GSE30656 
A

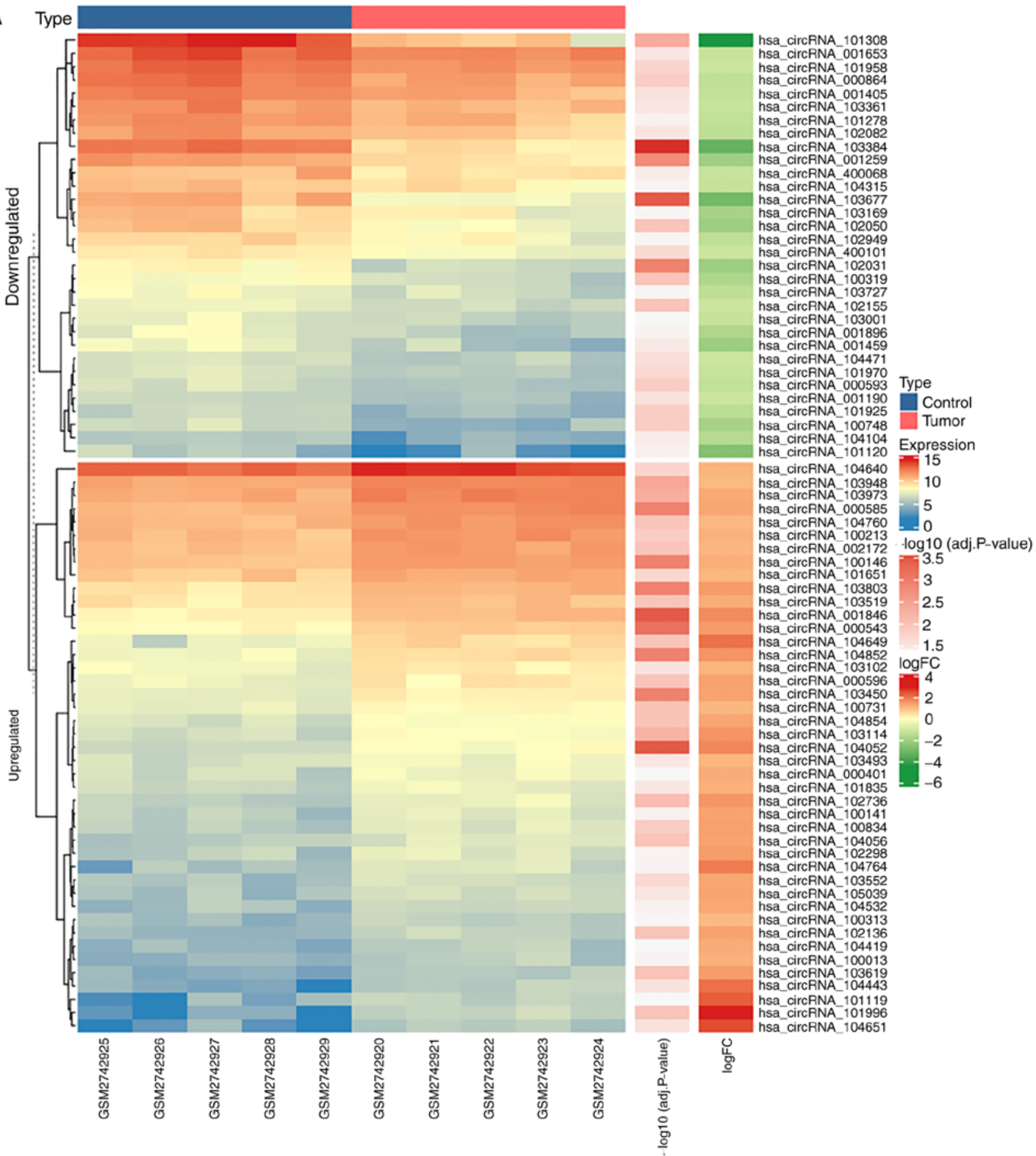

Figure 3. Expression of 75 DE-circRNAs and 5 DE-miRNAs in GEO. (A) DE-circRNAs from GSE102686.

is indicated in Fig. 3B. After integrating the DE-mRNAs from GSE63514 and CESC with the targeted genes from miRWalk, 155 DE-mRNAs were identified (Fig. 4A). The expression and oncoprint of these 155 genes in GSE63514 and TCGA is indicated in Fig. 4B.

Construction of miRNA-mRNA pairs. By merging the DE-mRNAs of GSE63514 and TCGA with the targeted mRNAs of 5 DE-miRNAs from miRWalk, 266 miRNA-mRNA pairs were selected. Specifically, 72 mRNAs of hsa-miR-15b, 16 mRNAs of hsa-miR-21, 2 mRNAs of hsa-miR-99a, 78 mRNAs of hsa-miR-106b and 58 mRNAs of hsa-miR-203 were obtained.
Construction of circRNA-miRNA pairs. Using RNAhybrid with the criterion that the circRNAs had perfect nucleotide pairing between the 2 nd and 8 th positions of the $5^{\prime}$ end of miRNA sequences, 12 circRNA-miRNA pairs were obtained.

Reconstruction of the circRNA-miRNA-mRNA network. As indicated in Fig. 5A, by combining the pairs of miRNA-mRNA and miRNA-circRNA, a preliminary circRNA-miRNA-mRNA network was constructed. The network was composed of 11 circRNA nodes, 4 miRNA nodes, 153 mRNA nodes and 203 edges. The network presented an initial perception of the association between the 11 DECs (hsa_circRNA_103102, hsa_circRNA_002172, hsa_circRNA_101835, 


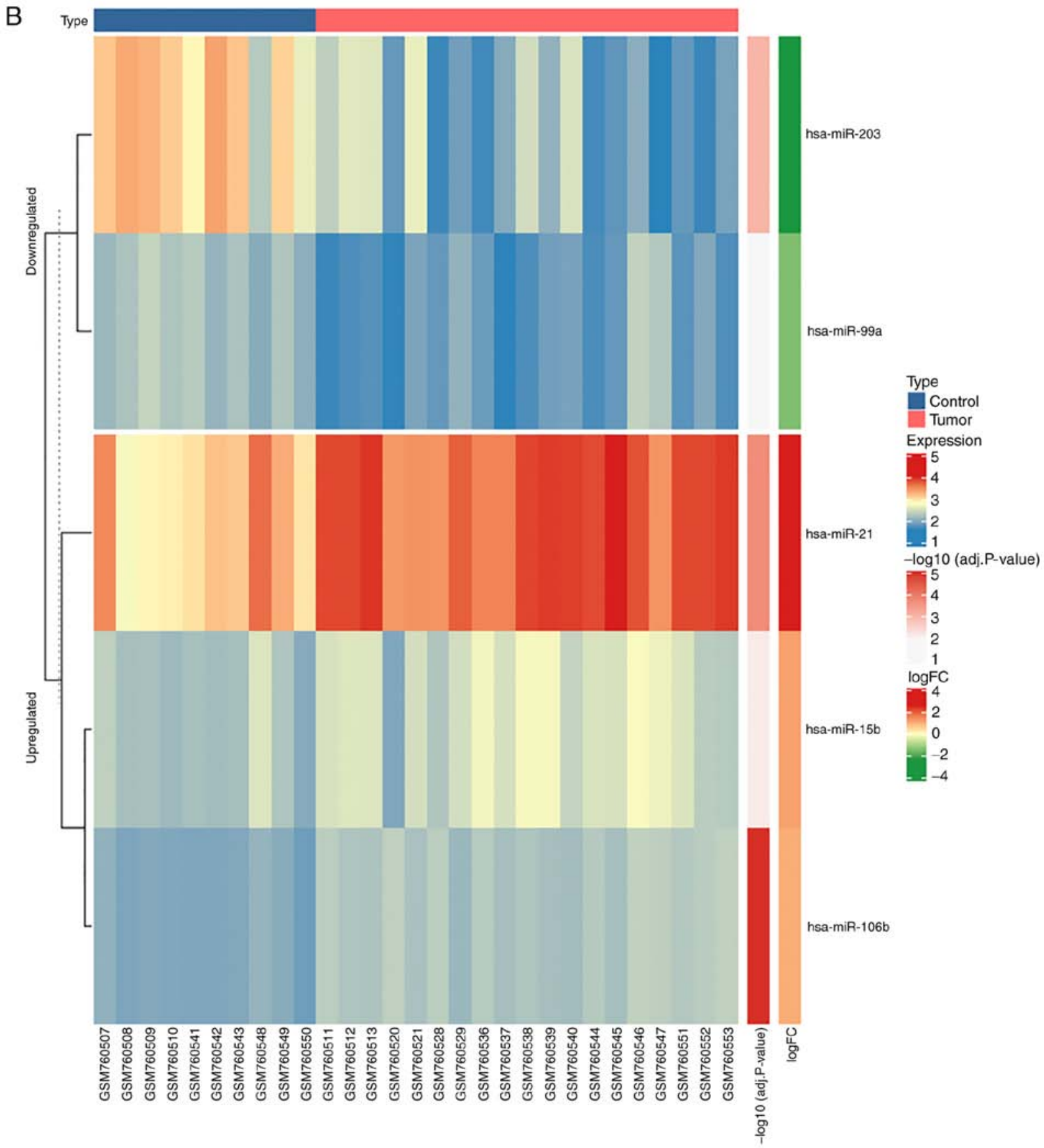

Figure 3. Continued. (B) DE-miRNAs from GSE306565. DE, differentially expressed; circRNA, circular RNA; miRNA, microRNA; GEO, Gene Expression Omnibus.

hsa_circRNA_102155, hs a_circRNA_102050, hsa_circRNA_103384, hsa_circRNA_104315, hsa_circRNA_400068, hsa_circRNA_000596, hsa_ circRNA_101958 and hsa_circRNA_103519), the 4 miRNAs (hsa-miR-21, hsa-miR-203, hsa-miR-15b and hsa-miR-106b) and the 153 mRNAs.

PPI network analysis. The STRING database was used to construct a PPI network based on the 155 overlapped mRNAs. The original network contained 47 nodes and 72 edges. By utilizing the algorithm of MCODE, four clusters were identified (Fig. 5B). Using the criteria of MCODE scores $>5$ and $>5$ nodes, one cluster was selected. There were 7 genes (RRM2,
CEP55, CHEK1, KIF23, RACGAP1, ATAD2 and KIF11) in this cluster, and these 7 genes were identified as hubgenes.

Construction of the circRNA-miRNA-hubgenes network. By mapping the 7 hubgenes into the preliminary circRNA-miRNA-mRNA network, and also extracting relevant circRNAs and miRNAs, a subnetwork considered as circRNA-miRNA-hubgenes was constructed. This subnetwork consisted of 5 circRNAs (hsa_circRNA_000596, hsa_circRNA_104315, hsa_circRNA_400068, hsa_circRNA_101958 and hsa_circRNA_103519), 2 mRNAs (hsa-miR-15b and hsa-miR-106b) and 7 mRNAs (RRM2, CEP55, CHEK1, KIF23, RACGAP1, 


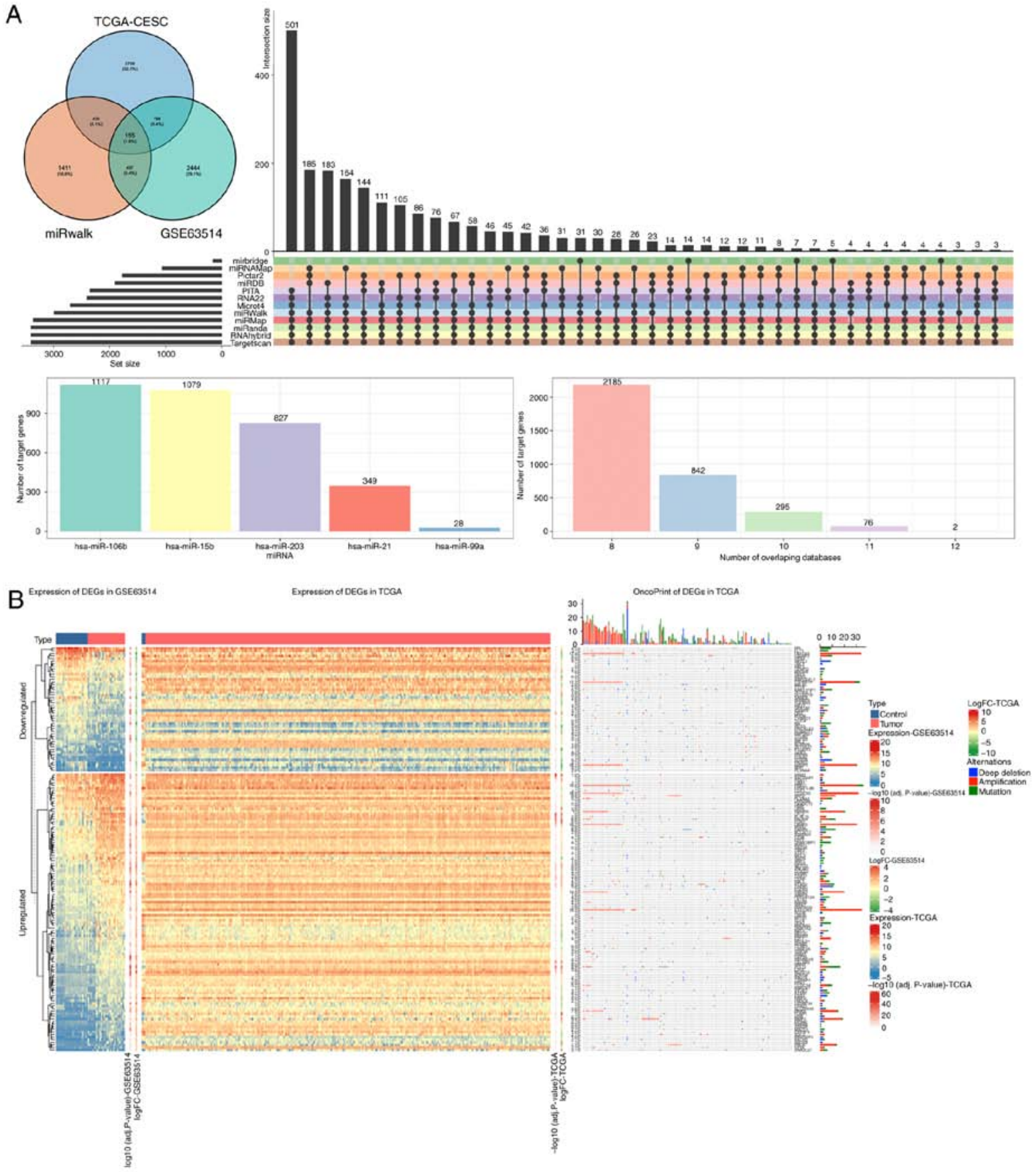

Figure 4. The 155 overlapped genes identified from GSE63514, TCGA and miRWalk with their expression and oncoprint. (A) UpsetR for miRWalk and Venn Plot for the 155 overlapped genes; and (B) expression and oncoprint of the 155 overlapped genes in GSE63514 and TCGA. TCGA, The Cancer Genome Atlas.

ATAD2 and KIF11) with 14 edges (Fig. 5C). There were 22 circRNA-miRNA-mRNA regulatory axes identified in the subnetwork (Table II). The structure and the primary characteristics of the circRNAs in the subnetwork were indicated in Fig. 6 and Table III. In addition, the stem-loop structure of hsa-miR-15b and hsa-miR-106b was revealed (Fig. 7A). The binding site to the circRNAs was also indicated (Fig. 7B).

GO and pathway enrichment analysis. The 155 overlapped genes were utilized for GO and pathway enrichment analysis using DAVID. For GO analysis, when considering BPs, the DEGs were enriched in intracellular signaling cascade, cell cycle and protein amino acid phosphorylation. With regards to $\mathrm{CC}$, the top three enriched items were cell death, neuron projection and cytoskeleton. In terms of MF, transcription factor activity, transcription regulator activity and protein kinase activity were enriched for the first three places. Pathway enrichment analysis indicated that the top three significantly enriched pathways were pathways in cancer, cell cycle and prostate cancer (Fig. 8).

Evaluation of OS for hubgenes. GEPIAT was used to assess the OS for the 7 hubgenes. Notably, higher expression of RRM2 revealed a significantly poorer OS (hazard ratio $=1.7$, $\mathrm{P}=0.029$ ). However, no significant effect was indicated for the remaining 6 hubgenes regarding OS (Fig. 9). 

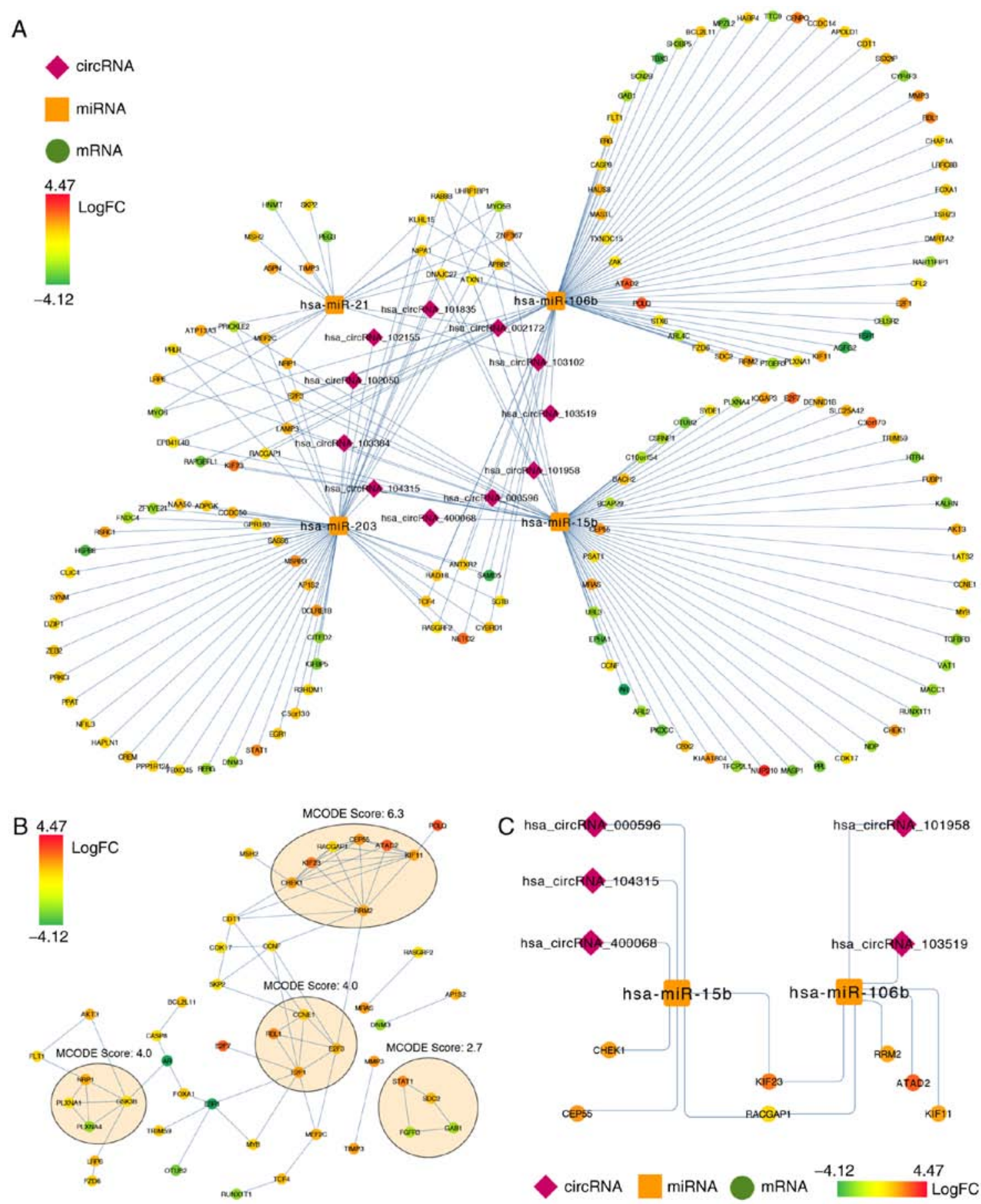

Figure 5. Preliminary circRNA-miRNA-mRNA, protein-protein interaction and circRNA-miRNA-hubgenes network for the 11 DECs (hsa_circRNA_103102, hsa_circRNA_002172, hsa_circRNA_101835, hsa_circRNA_102155, hsa_circRNA_102050, hsa_circRNA_103384, hsa_circRNA_104315, hsa_ circRNA_400068, hsa_circRNA_000596, hsa_circRNA_101958 and hsa_circRNA_103519), the 4 miRNAs (hsa-miR-21, hsa-miR-203, hsa-miR-15b and hsa-miR-106b) and the 155 mRNAs. (A) Preliminary circRNA-miRNA-mRNA network the 11 DECs, the 4 miRNAs and the 155 mRNAs. (B) Protein-protein interaction network for the 155 overlapped genes. Each ellipses represent the cluster identified by MCODE algorithm. (C) The circRNA-miRNA-hubgenes network for the 5 circRNAs (hsa_circRNA_000596, hsa_circRNA_104315, hsa_circRNA_400068, hsa_circRNA_101958 and hsa_circRNA_103519), the 2 mRNAs (hsa-miR-15b and hsa-miR-106b) and the 7 mRNAs (RRM2, CEP55, CHEK1, KIF23, RACGAP1, ATAD2 and KIF11). circRNA, circular RNA; miRNA/miR, microRNA; MCODE, Molecular Complex Detection.

Pharmacogenomics analysis for RRM2. As RRM2 significantly impacted OS, this gene was selected for the analysis of pharmacogenomics. Using PharmGkb, the SNPs of rs5030743 and rs1130609 were identified, which can be treated with cladribine and cytarabine (Table IV). Notably, cladribine is used for hairy cell leukemia, acute myeloid leukemia and chronic lymphocytic leukemia (41-43) while cytarabine is a chemotherapeutic drug utilized for various types of cancer, such as breast and lung cancer $(44,45)$. Cytarabine, similar to gemcitabine, is activated and metabolized by deoxycytidine kinase and cytidine deaminase (46). In addition, their primary metabolite can also incorporate DNA into cells (47). Hence, the gemcitabine pathway was selected to investigate the mechanism of action of cytarabine through RRM2. As indicated in 
Table II. Regulatory axis identified from the circRNA-miRNA-hubgene network.

\begin{tabular}{|c|c|c|c|}
\hline Regulatory axis & circRNA & miRNA & Hubgene \\
\hline 1 & hsa_circRNA_000596 & hsa-miR-15b & CHEK1 \\
\hline 2 & hsa_circRNA_000596 & hsa-miR-15b & CEP55 \\
\hline 3 & hsa_circRNA_104315 & hsa-miR-15b & CHEK1 \\
\hline 4 & hsa_circRNA_104315 & hsa-miR-15b & CEP55 \\
\hline 5 & hsa_circRNA_400068 & hsa-miR-15b & CHEK1 \\
\hline 6 & hsa_circRNA_400068 & hsa-miR-15b & CEP55 \\
\hline 7 & hsa_circRNA_000596 & hsa-miR-15b & KIF23 \\
\hline 8 & hsa_circRNA_000596 & hsa-miR-15b & RACGAP1 \\
\hline 9 & hsa_circRNA_104315 & hsa-miR-15b & KIF23 \\
\hline 10 & hsa_circRNA_104315 & hsa-miR-15b & RACGAP1 \\
\hline 11 & hsa_circRNA_400068 & hsa-miR-15b & KIF23 \\
\hline 12 & hsa_circRNA_400068 & hsa-miR-15b & RACGAP1 \\
\hline 13 & hsa_circRNA_101958 & hsa-miR-106b & KIF23 \\
\hline 14 & hsa_circRNA_101958 & hsa-miR-106b & RACGAP1 \\
\hline 15 & hsa_circRNA_103519 & hsa-miR-106b & KIF23 \\
\hline 16 & hsa_circRNA_103519 & hsa-miR-106b & RACGAP1 \\
\hline 17 & hsa_circRNA_101958 & hsa-miR-106b & RRM2 \\
\hline 18 & hsa_circRNA_103519 & hsa-miR-106b & RRM2 \\
\hline 19 & hsa_circRNA_101958 & hsa-miR-106b & ATAD2 \\
\hline 20 & hsa_circRNA_103519 & hsa-miR-106b & ATAD2 \\
\hline 21 & hsa_circRNA_101958 & hsa-miR-106b & KIF11 \\
\hline 22 & hsa_circRNA_103519 & hsa-miR-106b & KIF11 \\
\hline
\end{tabular}

miRNA, microRNA; circRNA, circular RNA.

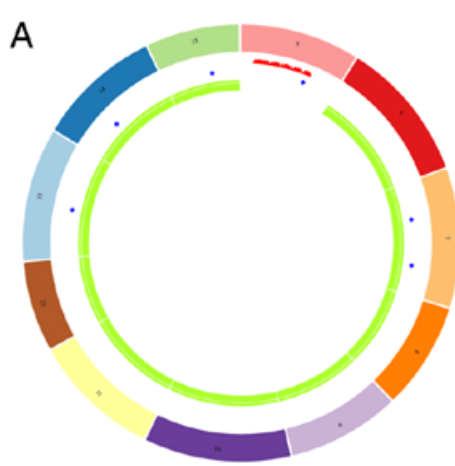

hsa_circRNA_000596

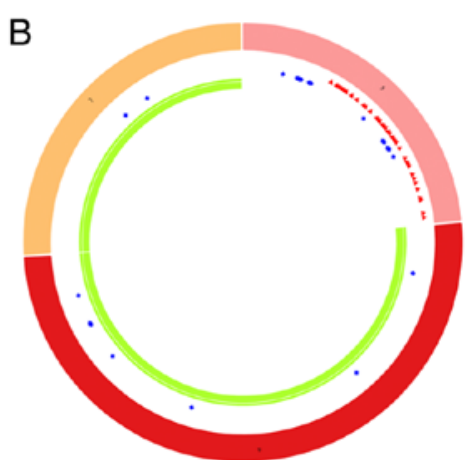

hsa_circRNA_400068

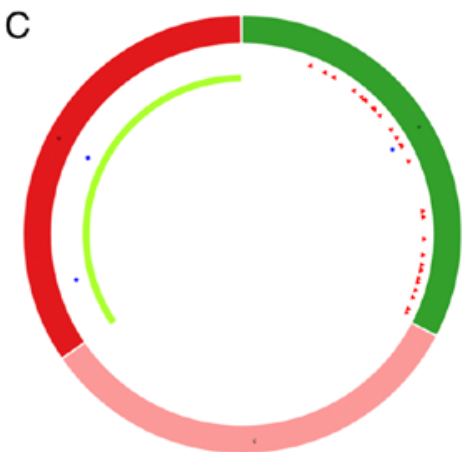

hsa_circRNA_104315

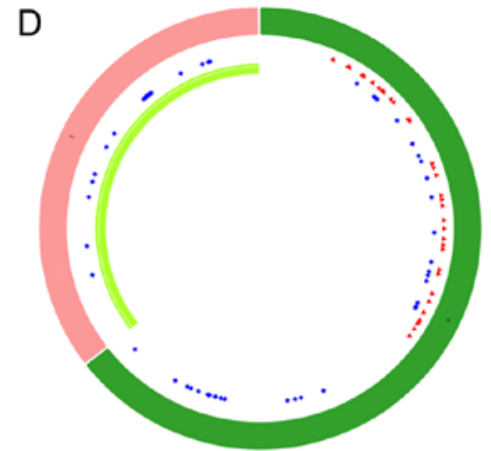

hsa_circRNA_101958
$\mathrm{E}$

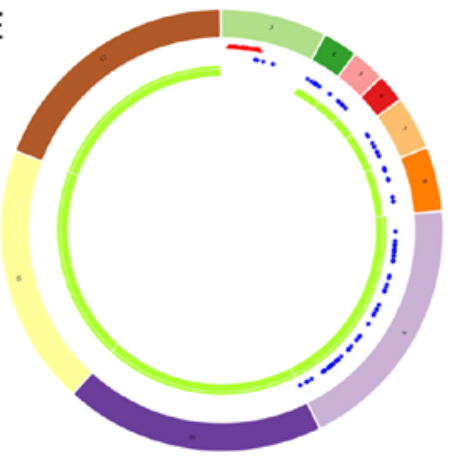

hsa_circRNA_103519

Figure 6. Structure of the 5 circRNAs predicted by the cancer-specific CircRNA. (A) hsa_circRNA_000596; (B) hsa_circRNA_400068; (C) hsa_ circRNA_104315; (D) hsa_circRNA_101958; (E) hsa_circRNA_103519. The different colors in the outer and inner ring represent the different exons and the number/position of MRE, RBP and ORF. MRE, microRNA response element; RBP, RNA binding protein; ORF, open reading frame; circRNA, circular RNA. 
Table III. Primary characteristics of the 5 circRNAs identified from the circRNA-miRNA-hubgene network.

\begin{tabular}{lcrrrrll}
\hline circRNA & Chromosome & Start position & End position & Strand & Location & Gene symbol & Regulation \\
\hline hsa_circRNA_000596 & Chr15 & 100589061 & 100739628 & + & Exon & ADAMTS17 & Upregulated \\
hsa_circRNA_400068 & Chr22 & 20109884 & 20114047 & + & Exon & RANBP1 & Downregulated \\
hsa_circRNA_104315 & Chr7 & 16298014 & 16330476 & - & Exon & ISPD & Downregulated \\
hsa_circRNA_101958 & Chr17 & 4192524 & 4207021 & - & Exon & UBE2G1 & Downregulated \\
hsa_circRNA_103519 & Chr3 & 179042885 & 179051613 & + & Exon & ZNF639 & Upregulated \\
\hline
\end{tabular}

miRNA, microRNA; circRNA, circular RNA.

A

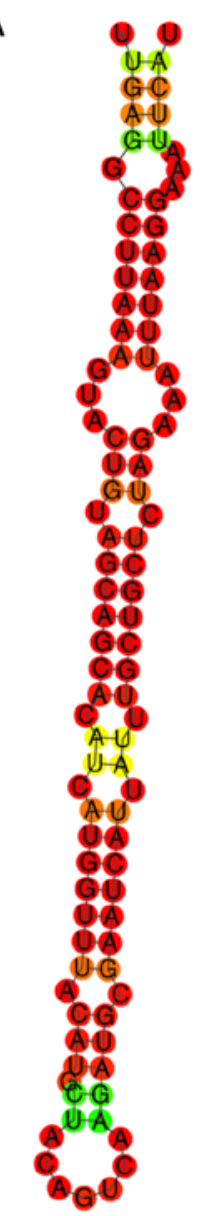

hsa-miR-15b

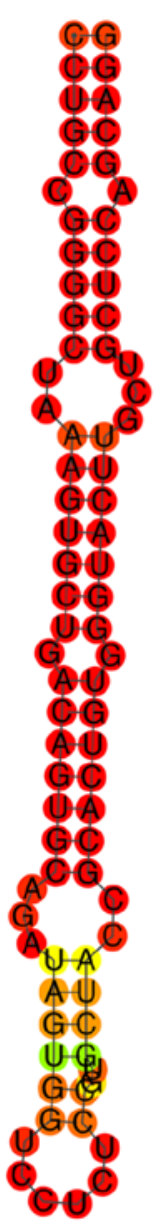

hsa-miR-106b

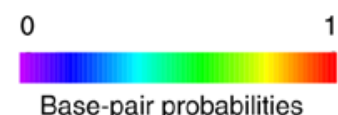

B
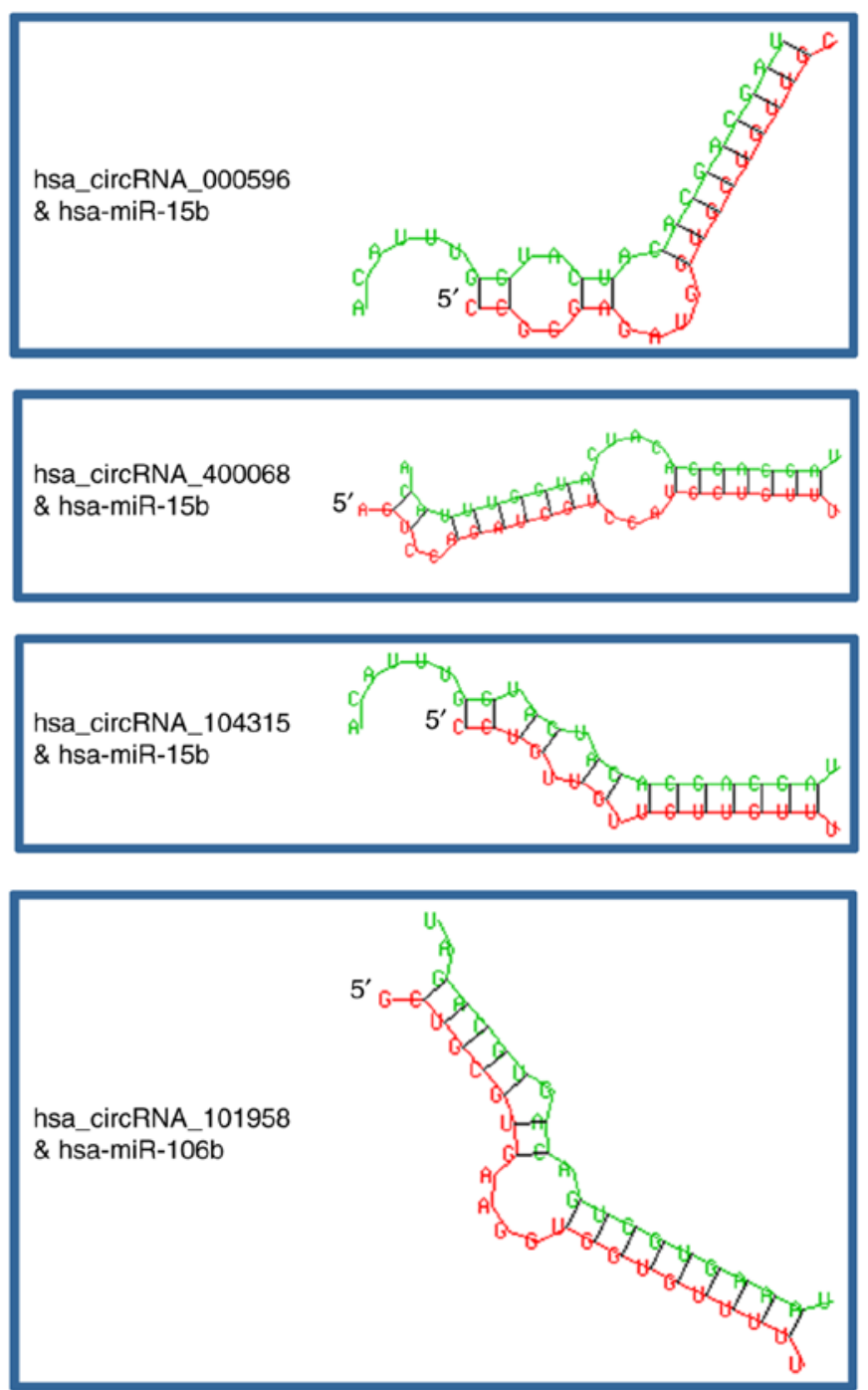

hsa_circRNA_103519

\& hsa-miR-106b

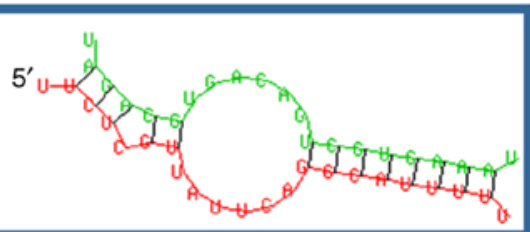

miRNA

Figure 7. Predicted stem-loop structure of hsa-miR-15b and has-miR-106b with the binding site to the corresponding circRNAs. (A) Predicted secondary stem-loop structure of hsa-miR-15b and has-miR-106b. The colors in each base pair represent its probabilities. (B) Binding site between hsa-miR-15b, has-miR-106b, hsa_circRNA_000596, hsa_circRNA_104315, hsa_circRNA_400068, hsa_circRNA_101958 and hsa_circRNA_103519. miRNA/miR, microRNA; circRNA, circular RNA. 

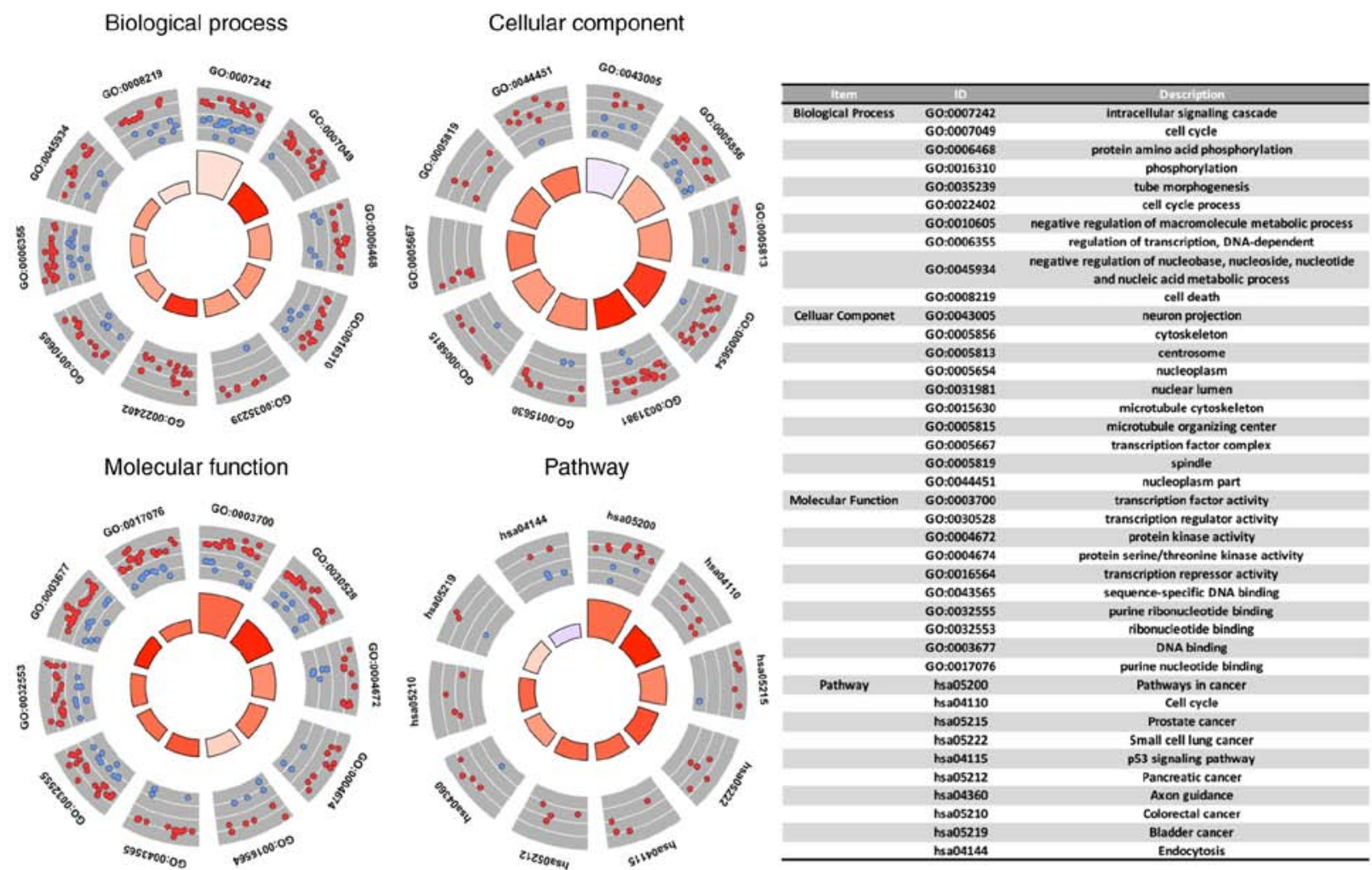

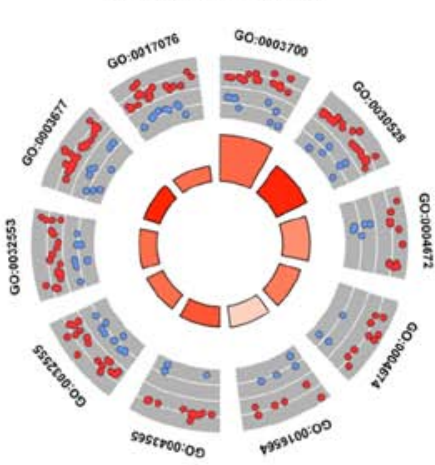

z-score

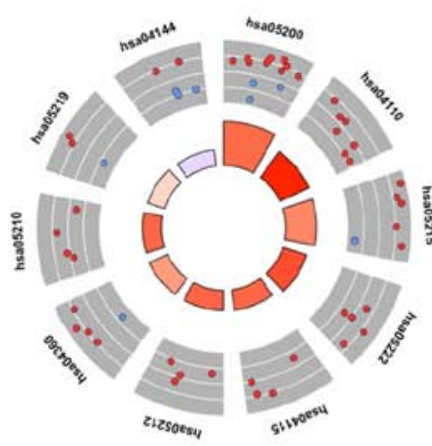

LogFC

Decreasing Increasing

- Downregulated $\bullet$ Upregulated

Figure 8. Top 10 items of biological process, cellular component and molecular function in Gene Ontology and pathway enrichment analysis for the 155 overlapped genes.
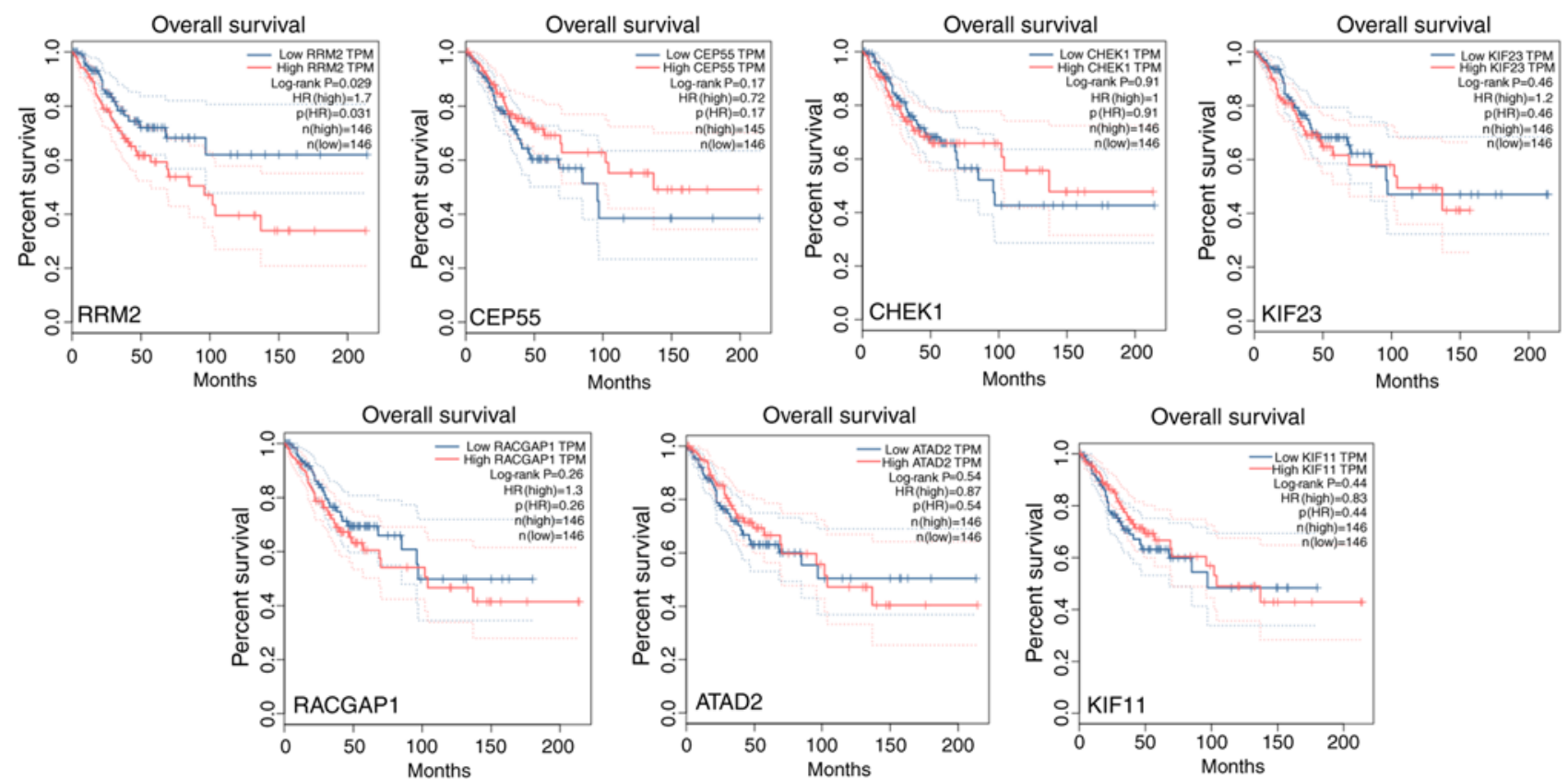

Figure 9. Overall survival for the 7 hubgenes (RRM2, CEP55, CHEK1, KIF23, RACGAP1, ATAD2 and KIF11) from TCGA. TPM, transcripts per million; HR, hazard ratio; TCGA, The Cancer Genome Atlas.

Fig. 10, gemcitabine produces its therapeutic effect by acting on the enzymes of RRM1, RRM2 and RRM2B, while the
RRM1, RRM2 and RRM2B genes also strictly regulate the activity of these enzymes. 


\section{Discussion}

During the past few decades, cervical cancer research has developed rapidly, particularly with regard to the discovery of the etiological factors. Although the key etiological role of HPV in the development of cervical cancer has been reported (48), the specific molecular mechanism remains unclear. Recently, more data has suggested that circRNAs participate in various biological processes (49-52). Dysregulated circRNA expression has been observed in the progression of complex diseases, including cervical cancer $(16,18,19,21)$. circRNAs are a novel class of competing endogenous ncRNAs that are widely expressed in eukaryotic cytoplasm (53). Unlike the RNAs formed by linear splicing, circRNAs have a closed loop structure with no 5' end cap and 3' end tail. The majority of known circRNAs are backspliced from exons, which are not easily degraded by exonuclease and have the feature of high abundance, structural stability and tissue specificity (54). With the rapid development of high-throughput RNA sequencing technology accompanied with the extensive data analysis by bioinformatics, circRNAs have been identified to possess the function of miRNA sponges (54-56), gene transcription regulation $(54,57)$ and RNA-binding proteins $(58-60)$ as well as their translation $(52,61,62)$. In addition, studies have confirmed that circRNA serves an important role in tumor cells, so may potentially serve as a novel biomarker and therapeutic target for cancer therapy (63-65). However, more circRNAs need to be uncovered. In the present study, the circRNA microarray profile of GSE102686 for cervical cancer was screened to identify DE-circRNAs. Using the criteria of FDR $<0.05$ and $\log \mid \mathrm{FCl}>1$ calculated by $\mathrm{R}$ with limma package, 75 DE-circRNAs were selected for further analysis.

Previous studies have indicated that circRNA is a type of high-efficiency ceRNA $(66,67)$. It can inhibit the binding of miRNAs to target genes and regulate the expression level of target genes by exerting a miRNA sequestering effect (68). To determine whether the above 75 circRNAs function as ceRNAs in cervical cancer, the targeted miRNAs with the sequence of DE-circRNAs and DE-miRNAs were predicted with RNAhybrid. Furthermore, 12 circRNA-miRNA pairs were identified. hsa-miR-106b, hsa-miR-15b and hsa-miR-21 were identified to be upregulated in CESC and GSE30656, and these results were consistent with previous findings (69-73). Notably, hsa-miR-203 was upregulated in CESC but downregulated in GSE30656. Previous studies have suggested that the expression of miR-203 is typically downregulated in cervical cancer tumors and cell lines (74-76). This finding is in line with the expression profile of GSE30656 but contradicts to TCGA. Reshmi and Pillai (77) also identified that miR-203 can be overexpressed or underexpressed in cervical cancer cell lines. Furthermore, Zhao et al (78) indicated that the expression level of miR-203 in serum of patients with cervical cancer was significantly upregulated; however, miR-203 downregulation was correlated with lymph nodes metastasis. Hence, it was possible that upregulation and downregulation of miR-203 could promote the progression of cervical cancer but serves different functions. Upregulation of miR-203 could also trigger the occurrence of cervical cancer whereas downregulation may enhance the metastatic capacity of lymph nodes. However, the specific mechanism requires further investigation. 


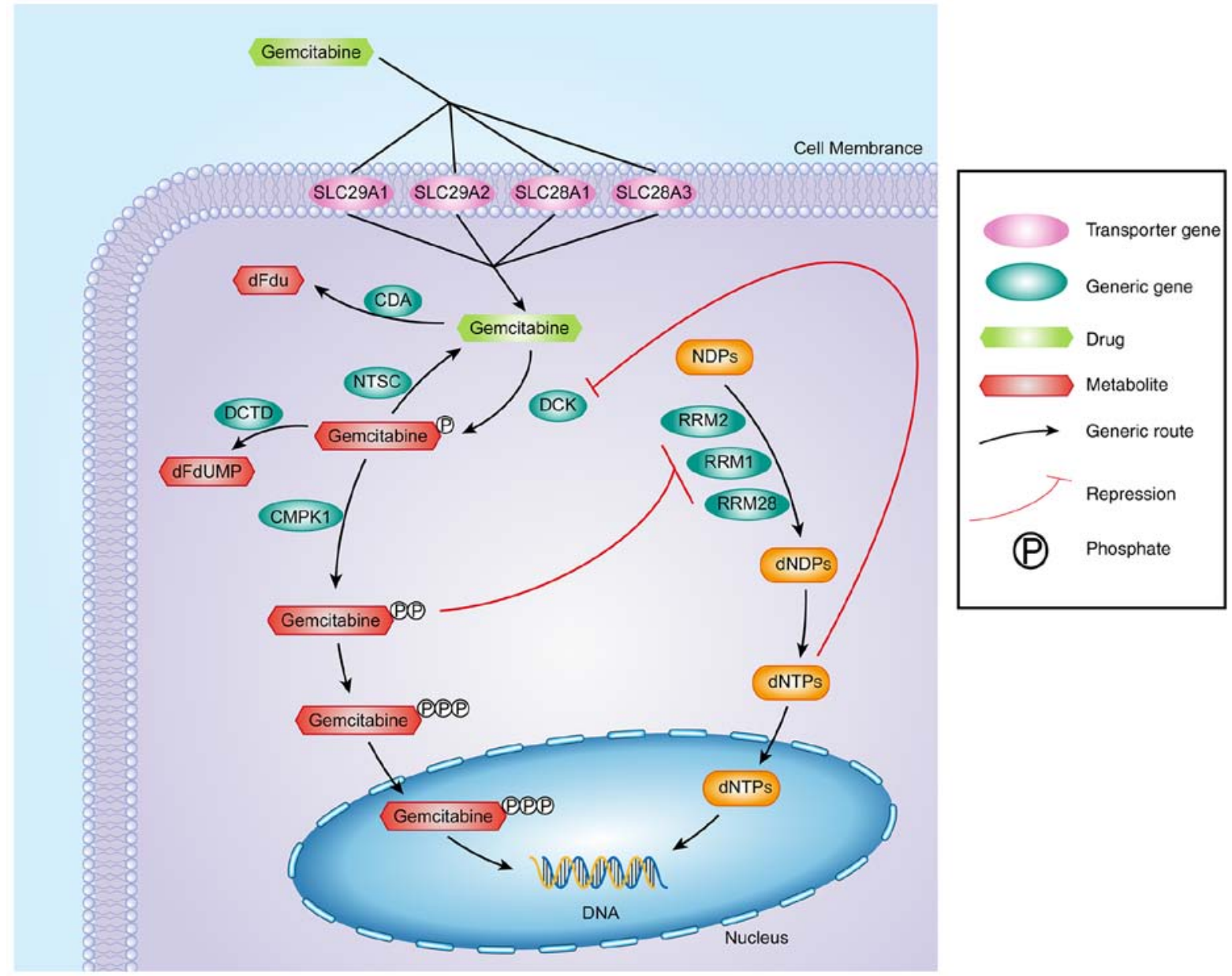

Figure 10. Gemcitabine pathway extracted from PharmGkb database. Gemcitabine could exert its therapeutic effect by acting on RRM2, RRM1 and RRM2B. dFdu, 2',2'-difluorodeoxyuridine; dFdUMP, dFdU monophosphate; NDP, nucleoside diphosphates; dNDP, deoxy-ribonucleoside diphosphate; dNTP, deoxy-ribonucleoside triphosphate.

Once the 155 overlapping genes with the DE-circRNAs and DE-miRNAs were collected, a circRNA-miRNA-mRNA regulatory network was reconstructed. The 11 circRNAs could bind to hsa-miR-21, hsa-miR-203, hsa-miR-106b or hsa-miR-15b as ceRNAs to regulate the expression of 155 genes. The present results provide evidence for the ceRNA regulatory mechanisms of 11 circRNAs in cervical cancer. The GO and pathway enrichment analysis indicated that 155 genes were involved in various important biological functions and metabolic pathways associated with tumors, including 'Pathways in cancer', 'Cell cycle', 'Prostate cancer', 'Small cell lung cancer', 'p53 signaling pathway' and 'Pancreatic cancer'. It also indicated that several type of cancer, such as prostate cancer, lung cancer and pancreatic cancer, share the same pathways with cervical cancer, and provided evidence for the research of pan-cancer. To further understand the functional mechanism of the ceRNA-miRNA-mRNA network, the PPI network was constructed and 7 hubgenes (RRM2, CEP55, CHEK1, KIF23, RACGAP1, ATAD2 and KIF11) were identified from the PPI network. The crucial mechanisms of RRM2, CEP55, CHEK1, ATAD2 and KIF11 in cervical cancer have been previously studied (79-82). However, to the best of our knowledge, KIF23 and RACGAP1 have not yet been investigated. The 22 circRNA-miRNA-mRNA axes, which were identified from the circRNA-miRNA-hubgenes, revealed the competing regulatory associations between the 5 circRNAs and the 2 mRNAs with the 7 hubgenes in cervical cancer. However, as the present study was an in silico research, a further experiment of these 22 regulatory axes is required for validation.

The OS for the 7 hubgenes was evaluated in the present study and it was revealed that RRM2 had a significant effect on OS. Subsequently, RRM2 was investigated using the database of PharmGkb. It was revealed that cytarabine, which is similar to gemcitabine, may produce its therapeutic effect by targeting to the SNPs of rs5030743 and rs1130609 of RRM2 through the gemcitabine pathway. To date, gemcitabine has been indicated to be feasible and effective on cervical cancer (83-85). However, cytarabine has not yet been investigated in cervical cancer, nor the rs5030743 and rs1130609 of RRM2. Although cytarabine differs from gemcitabine in several important respects (86), for instance, gemcitabine differs structurally from cytarabine by a fluorine group substituted at position $2^{\prime}$ on the furanose ring (87), gemcitabine (88-90) and cytarabine $(91,92)$ can be a treatment for breast cancer. Hence, cytarabine may produce a similar effect to gemcitabine and could be an optional chemotherapeutic drug for treating cervical cancer by targeting rs5030743 and rs 1130609 or other similar SNPs. However, further study is necessary to validate the hypothesis. 
To conclude, the present study constructed and analyzed a circRNA-miRNA-mRNA network based on the ceRNA theory via comprehensive bioinformatics analysis, which may provide some evidence to future studies focused on the molecular mechanisms of cervical cancer. The 5 circRNAs (hsa_circRNA_000596, hsa_circRNA_104315, hsa_circRNA_400068, hsa_circRNA_101958 and hsa_ circRNA_103519) may function as ceRNAs to serve critical roles in cervical cancer. In addition, cytarabine may produce a similar effect to gemcitabine and could be an optional chemotherapeutic drug for cervical cancer by targeting rs5030743 and rs1130609 or other similar SNPs. However, the specific mechanism of action should be confirmed by further study.

\section{Acknowledgements}

Not applicable.

\section{Funding}

No funding was received.

\section{Availability of data and materials}

All data generated or analyzed during this study are included in this published article.

\section{Authors' contributions}

YY and YL established the concept of the present study and drafted the paper. WW and KW performed the literature research, data extraction and statistical analysis. WZ contributed in the performing the literature research, data extraction and analysis, and reviewed and approved the final version of the paper. All authors have read and approved the manuscript.

\section{Ethics approval and consent to participate}

The study protocol was approved by the Ethics Committee of Zhongnan Hospital of Wuhan University. Informed consent forms were signed by all participants.

\section{Patient consent for publication}

Not applicable.

\section{Competing interests}

The authors declare that they have no competing interests.

\section{References}

1. Torre LA, Bray F, Siegel RL, Ferlay J, Lortet-Tieulent J and Jemal A: Global cancer statistics, 2012. CA Cancer J Clin 65: 87-108, 2015.

2. Marth C, Landoni F, Mahner S, McCormack M, GonzalezMartin A and Colombo N; ESMO Guidelines Committee: Cervical cancer: ESMO Clinical Practice Guidelines for diagnosis, treatment and follow-up. Ann Oncol 28 (Suppl 4): iv72-iv83, 2017.
3. GuYu Z, YiMin Z, ChongDong L, GuangMing C, Ran C and ZhenYu Z: Current status and future of targeted therapy for patients with local advanced cervical cancer. Chinese J Pract Gynecol Obstet 34: 1216-1220, 2018.

4. Fleming ND, Frumovitz M, Schmeler KM, dos Reis R, Munsell MF, Eifel PJ, Soliman PT, Nick AM, Westin SN and Ramirez PT: Significance of lymph node ratio in defining risk category in node-positive early stage cervical cancer. Gynecol Oncol 136: 48-53, 2015.

5. Kapranov P, Cawley SE, Drenkow J, Bekiranov S, Strausberg RL, Fodor SP and Gingeras TR: Large-scale transcriptional activity in chromosomes 21 and 22. Science 296: 916-919, 2002.

6. Esteller M: Non-coding RNAs in human disease. Nat Rev Genet 12: 861-874, 2011.

7. Kogo R, How C, Chaudary N, Bruce J, Shi W, Hill RP, Zahedi P, Yip KW and Liu FF: The microRNA-218 Survivin axis regulates migration, invasion, and lymph node metastasis in cervical cancer. Oncotarget 6: 1090-1100, 2014.

8. Hou T, Ou J, Zhao X, Huang X, Huang Y and Zhang Y: MicroRNA-196a promotes cervical cancer proliferation through the regulation of FOXO1 and p27 ${ }^{\mathrm{Kip} 1}$. Br J Cancer 110: 1260-1268, 2014.

9. Wang Q, Qin J, Chen A, Zhou J, Liu J, Cheng J, Qiu J and Zhang J: Downregulation of microRNA-145 is associated with aggressive progression and poor prognosis in human cervical cancer. Tumor Biol 36: 3703-3708, 2015 .

10. Fang H, Shuang D, Yi Z, Sheng H and Liu Y: Up-regulated microRNA-155 expression is associated with poor prognosis in cervical cancer patients. Biomed Pharmacother 83: 64-69, 2016.

11. Bumrungthai S, Ekalaksananan T, Evans MF, Chopjitt P, Tangsiriwatthana T, Patarapadungkit N, Kleebkaow $\mathrm{P}$, Luanratanakorn S, Kongyingyoes B, Worawichawong S, et al: Up-regulation of miR-21 is associated with cervicitis and human papillomavirus infection in cervical tissues. PLoS One 10: e0127109, 2015.

12. Salmena L, Poliseno L, Tay Y, Kats L and Pandolfi PP: A ceRNA hypothesis: The Rosetta Stone of a hidden RNA language? Cell 146: 353-8, 2011.

13. Sanger HL, Klotz G, Riesner D, Gross HJ and Kleinschmidt AK: Viroids are single-stranded covalently closed circular RNA molecules existing as highly base-paired rod-like structures. Proc Natl Acad Sci USA 73: 3852-3856, 1976.

14. Memczak S, Jens M, Elefsinioti A, Torti F, Krueger J, Rybak A, Maier L, Mackowiak SD, Gregersen LH, Munschauer M, et al: Circular RNAs are a large class of animal RNAs with regulatory potency. Nature 495: 333-338, 2013.

15. Hansen TB, Jensen TI, Clausen BH, Bramsen JB, Finsen B, Damgaard CK and Kjems J: Natural RNA circles function as efficient microRNA sponges. Nature 495: 384-388, 2013.

16. Tian M, Chen R, Li T and Xiao B: Reduced expression of circRNA hsa_circ_0003159 in gastric cancer and its clinical significance. J Clin Lab Anal 32, 2018.

17. Liu Q, Zhang X, Hu X, Yuan L, Cheng J, Jiang Y and Ao Y: Emerging roles of circRNA related to the mechanical stress in human cartilage degradation of osteoarthritis. Mol Ther Nucleic Acids 7: 223-230, 2017.

18. Huang XY, Huang ZL, Xu YH,Zheng Q, Chen Z, Song W, Zhou J, Tang ZY and Huang XY: Comprehensive circular RNA profiling reveals the regulatory role of the circRNA-100338/MIR-141-3p pathway in hepatitis B-related hepatocellular carcinoma. Sci Rep 7: 5428, 2017.

19. Hsiao KY, Lin YC, Gupta SK, Chang N, Yen L, Sun HS and Tsai SJ: Noncoding effects of circular RNA CCDC66 promote colon cancer growth and metastasis. Cancer Res 77: 2339-2350, 2017.

20. Wang Y, Huang L, Li D, Shao J, Xiong S, Wang C and Lu S: Hsa_circ_0101996 combined with hsa_circ_0101119 in peripheral whole blood can serve as the potential biomarkers for human cervical squamous cell carcinoma. Int J Clin Exp Pathol 10: 11924-11931, 2017.

21. Gao YL, Zhang MY, Xu B, Han LJ, Lan SF, Chen J, Dong YJ and Cao LL: Circular RNA expression profiles reveal that hsa circ_0018289 is up-regulated in cervical cancer and promotes the tumorigenesis. Oncotarget 8: 86625-86633, 2017.

22. Ma HB, Yao YN, Yu JJ, Chen XX and Li HF: Extensive profiling of circular RNAs and the potential regulatory role of circRNA-000284 in cell proliferation and invasion of cervical cancer via sponging miR-506. Am J Transl Res 10: 592-604, 2018. 
23. Barrett T, Wilhite SE, Ledoux P, Evangelista C, Kim IF Tomashevsky M, Marshall KA, Phillippy KH, Sherman PM, Holko M, et al: NCBI GEO: Archive for functional genomics data sets-update. Nucleic Acids Res 41: D991-D995, 2013.

24. Gao J, Aksoy BA, Dogrusoz U, Dresdner G, Gross B, Sumer SO, Sun Y, Jacobsen A, Sinha R, Larsson E, et al: Integrative analysis of complex cancer genomics and clinical profiles using the cBioPortal. Sci Signal 6: pl1, 2013.

25. Ritchie ME, Phipson B, Wu D, Hu Y, Law CW, Shi W and Smyth GK: limma powers differential expression analyses for RNA-sequencing and microarray studies. Nucleic Acids Res 43: e47, 2015 .

26. Dweep H and Gretz N: MiRWalk2.0: A comprehensive atlas of microRNA-target interactions. Nat Methods 12: 697, 2015.

27. Agarwal V, Bell GW, Nam JW and Bartel DP: Predicting effective microRNA target sites in mammalian mRNAs. Elife 4, 2015.

28. Pasquinelli AE: MicroRNAs and their targets: Recognition, regulation and an emerging reciprocal relationship. Nat Rev Genet 13: 271-282, 2012.

29. Conway JR, Lex A and Gehlenborg N: UpSetR: An R package for the visualization of intersecting sets and their properties. Bioinformatics 33: 2938-2940, 2017.

30. Kozomara A and Griffiths-Jones S: MiRBase: Annotating high confidence microRNAs using deep sequencing data. Nucleic Acids Res 42 (Database Issue): D68-D73, 2014.

31. Krüger J and Rehmsmeier M: RNAhybrid: MicroRNA target prediction easy, fast and flexible. Nucleic Acids Res 34: W451-W454, 2006.

32. Jiang H, Ma R, Zou S, Wang Y, Li Z and Li W: Reconstruction and analysis of the lncRNA-miRNA-mRNA network based on competitive endogenous RNA reveal functional lncRNAs in rheumatoid arthritis. Mol Biosyst 13: 1182-1192, 2017.

33. Shannon P, Markiel A, Ozier O, Baliga NS, Wang JT, Ramage D, Amin N, Schwikowski B and Ideker T: Cytoscape: A software environment for integrated models of biomolecular interaction networks. Genome Res 13: 2498-2504, 2003.

34. Jiao X, Sherman BT, Huang da W, Stephens R, Baseler MW, Lane HC and Lempicki RA: DAVID-WS: A stateful web service to facilitate gene/protein list analysis. Bioinformatics 28 : 1805-1806, 2012.

35. Walter W, Sánchez-Cabo F and Ricote M: GOplot: An R package for visually combining expression data with functional analysis. Bioinformatics 31: 2912-2914, 2015.

36. Szklarczyk D, Franceschini A, Wyder S, Forslund K, Heller D, Huerta-Cepas J, Simonovic M, Roth A, Santos A, Tsafou KP, et al STRING v10: Protein-protein interaction networks, integrated over the tree of life. Nucleic Acids Res 43: D447-D452, 2015.

37. Bader GD and Hogue CW: An automated method for finding molecular complexes in large protein interaction networks. BMC Bioinformatics 4: 2, 2003.

38. Xia S, Feng J, Chen K, Ma Y, Gong J, Cai F, Jin Y, Gao Y, Xia L, Chang H, et al: CSCD: A database for cancer-specific circular RNAs. Nucleic Acids Res 46: D925-D929, 2018.

39. Kerpedjiev P, Hammer S and Hofacker IL: Forna (force-directed RNA): Simple and effective online RNA secondary structure diagrams. Bioinformatics 31: 3377-3379, 2015.

40. Tang Z, Li C, Kang B, Gao G, Li C and Zhang Z: GEPIA: A web server for cancer and normal gene expression profiling and interactive analyses. Nucleic Acids Res 45: W98-W102, 2017.

41. Schoen MW, Woelich SK, Braun JT, Reddy DV, Fesler MJ, Petruska PJ, Freter CE and Lionberger JM: Acute myeloid leukemia induction with cladribine: Outcomes by age and leukemia risk. Leuk Res 68: 72-78, 2018.

42. Johnston JB: Mechanism of action of pentostatin and cladribine in hairy cell leukemia. Leuk Lymphoma 52 (Suppl 2): S43-S45, 2011.

43. Mulligan SP, Karlsson K, Strömberg M, Jønsson V, Gill D, Hammerström J, Hertzberg M, McLennan R, Uggla B Norman J, et al: Cladribine prolongs progression-free survival and time to second treatment compared to fludarabine and high-dose chlorambucil in chronic lymphocytic leukemia. Leuk Lymphoma 55: 2769-2777, 2014.

44. Mego M, Sycova-Mila Z, Obertova J, Rajec J, Liskova S, Palacka P, Porsok S and Mardiak J: Intrathecal administration of trastuzumab with cytarabine and methotrexate in breast cancer patients with leptomeningeal carcinomatosis. Breast 20: 478-480, 2011
45. Rusch VW, Figlin R, Godwin D and Piantadosi S: Intrapleural cisplatin and cytarabine in the management of malignant pleural effusions: A Lung Cancer Study Group trial. J Clin Oncol 9: 313-319, 1991.

46. Baker JAR, Wickremsinhe ER, Li CH, Oluyedun OA, Dantzig AH, Hall SD, Qian YW, Ring BJ, Wrighton SA and Guo Y: Pharmacogenomics of gemcitabine metabolism: Functional analysis of genetic variants in cytidine deaminase and deoxycytidine kinase. Drug Metab Dispos 41: 541-545, 2013.

47. Lamba JK: Genetic factors influencing cytarabine therapy. Pharmacogenomics 10: 1657-1674, 2009.

48. Walboomers JM, Jacobs MV, Manos MM, Bosch FX, Kummer JA, Shah KV, Snijders PJ, Peto J, Meijer CJ and Muñoz N: Human papillomavirus is a necessary cause of invasive cervical cancer worldwide. J Pathol 189: 12-19, 1999.

49. Rong D, Sun H, Li Z, Liu S, Dong C, Fu K, Tang W and Cao H: An emerging function of circRNA-miRNAs-mRNA axis in human diseases. Oncotarget 8: 73271-73281, 2017.

50. Du WW, Zhang C, Yang W, Yong T, Awan FM and Yang BB: Identifying and characterizing circRNA-protein interaction. Theranostics 7: 4183-4191, 2017.

51. Ebbesen KK, Hansen TB and Kjems J: Insights into circular RNA biology. RNA Biol 14: 1035-1045, 2017.

52. Pamudurti NR, Bartok O, Jens M, Ashwal-Fluss R, Stottmeister C, Ruhe L, Hanan M, Wyler E, Perez-Hernandez D, Ramberger E, et al: Translation of CircRNAs. Mol Cell 66: 9-21. e7, 2017.

53. Holdt LM, Kohlmaier A and Teupser D: Molecular roles and function of circular RNAs in eukaryotic cells. Cell Mol Life Sci 75: 1071-1098, 2018.

54. Jeck WR, Sorrentino JA, Wang K, Slevin MK, Burd CE, Liu J, Marzluff WF and Sharpless NE: Circular RNAs are abundant, conserved, and associated with ALU repeats. RNA 19: 141-57, 2013.

55. Xie B, Zhao Z, Liu Q, Wang X, Ma Z and Li H: CircRNA has_circ_0078710 acts as the sponge of microRNA-31 involved in hepatocellular carcinoma progression. Gene 683: 253-261, 2019.

56. Xiong D, Dang Y, Lin P, Wen DY, He RQ, Luo DZ, Feng ZB and Chen G: A circRNA-miRNA-mRNA network identification for exploring underlying pathogenesis and therapy strategy of hepatocellular carcinoma. J Transl Med 16: 220, 2018.

57. Salzman J, Chen RE, Olsen MN, Wang PL and Brown PO: Cell-type specific features of circular RNA expression. PLoS Genet 9: e1003777, 2013.

58. Zhang Y, Zhang XO, Chen T, Xiang JF, Yin QF, Xing YH, Zhu S, Yang $L$ and Chen LL: Circular intronic long noncoding RNAs. Mol Cell 51: 792-806, 2013.

59. Li Z, Huang C, Bao C, Chen L, Lin M, Wang X, Zhong G, Yu B, $\mathrm{Hu}$ W, Dai L, et al: Exon-intron circular RNAs regulate transcription in the nucleus. Nat Struct Mol Biol 22: 256-264, 2015.

60. You X, Vlatkovic I, Babic A, Will T, Epstein I, Tushev G, Akbalik G, Wang M, Glock C, Quedenau C, et al: Neural circular RNAs are derived from synaptic genes and regulated by development and plasticity. Nat Neurosci 18: 603-610, 2015.

61. Legnini I, Di Timoteo G, Rossi F, Morlando M, Briganti F, Sthandier O, Fatica A, Santini T, Andronache A, Wade M, et al: Circ-ZNF609 is a circular RNA that can be translated and functions in myogenesis. Mol Cell 66: 22-37.e9, 2017.

62. Yang Y, Fan X, Mao M, Song X, Wu P, Zhang Y, Jin Y, Yang Y, Chen LL, Wang Y, et al: Extensive translation of circular RNAs driven by N6-methyladenosine. Cell Res 27: 626-641, 2017.

63. Li F, Zhang L, Li W, Deng J, Zheng J, An M, Lu J and Zhou Y: Circular RNA ITCH has inhibitory effect on ESCC by suppressing the Wnt/ $\beta$-catenin pathway. Oncotarget 6: 6001-6013, 2015.

64. Xie H, Ren X, Xin S, Lan X, Lu G, Lin Y, Yang S, Zeng Z, Liao W, Ding YQ and Liang L: Emerging roles of circRNA 001569 targeting miR-145 in the proliferation and invasion of colorectal cancer. Oncotarget 7: 26680-26691, 2016.

65. Li P, Chen S, Chen H, Mo X, Li T, Shao Y, Xiao B and Guo J: Using circular RNA as a novel type of biomarker in the screening of gastric cancer. Clin Chim Acta 444: 132-136, 2015.

66. Mitra A, Pfeifer K and Park KS: Circular RNAs and competing endogenous RNA (ceRNA) networks. Transl Cancer Res 7 (Suppl 5): S624-S628, 2018.

67. Holdt LM, Kohlmaier A and Teupser D: Molecular functions and specific roles of circRNAs in the cardiovascular system. Non-coding RNA Res 3: 75-98, 2018. 
68. Liu Q, Zhang X, Hu X, Dai L, Fu X, Zhang J and Ao Y: Circular RNA related to the chondrocyte ECM regulates MMP13 expression by functioning as a miR-136 'Sponge' in human cartilage degradation. Sci Rep 6: 22572, 2016.

69. Wang X, Tang S, Le SY, Lu R, Rader JS, Meyers C and Zheng ZM: Aberrant expression of oncogenic and tumor-suppressive microRNAs in cervical cancer is required for cancer cell growth PLoS One 3: e2557, 2008.

70. Li Y, Wang F, Xu J, Ye F, Shen Y, Zhou J, Lu W, Wan X, Ma D and Xie X: Progressive miRNA expression profiles in cervical carcinogenesis and identification of HPV-related target genes for miR-29. J Pathol 224: 484-495, 2011.

71. Yi Y, Liu Y, Wu W, Wu K and Zhang W: The role of miR-106p-5p in cervical cancer: From expression to molecular mechanism. Cell death Discov 4: 36, 2018.

72. Park S, Eom K, Kim J, Bang H, Wang HY, Ahn S, Kim G, Jang H, Kim S, Lee D, et al: MiR-9, miR-21, and miR-155 as potential biomarkers for HPV positive and negative cervical cancer. BMC Cancer 17: 658, 2017.

73. Xu L, Xu Q, Li X and Zhang X: MicroRNA-21 regulates the proliferation and apoptosis of cervical cancer cells via tumor necrosis factor- $\alpha$. Mol Med Rep 16: 4659-4663, 2017.

74. Zhu X, Er K, Mao C, Yan Q, Xu H, Zhang Y, Zhu J, Cui F, Zhao W and Shi H: miR-203 suppresses tumor growth and angiogenesis by targeting VEGFA in cervical cancer. Cell Physiol Biochem 32 64-73, 2013.

75. Melar-New M and Laimins LA: Human papillomaviruses modulate expression of MicroRNA 203 upon epithelial differentiation to control levels of p63 proteins. J Virol 84: 5212-5221, 2010.

76. Mao L, Zhang Y, Mo W, Yu Y and Lu H: BANF1 is downregulated by IRF1-regulated microRNA-203 in cervical cancer. PLoS One 10: e0117035, 2015.

77. Reshmi G and Pillai MR: Beyond HPV: Oncomirs as new players in cervical cancer. FEBS Lett 582: 4113-4116, 2008.

78. Zhao S, Yao D, Chen J and Ding N: Circulating miRNA-20a and miRNA-203 for screening lymph node metastasis in early stage cervical cancer. Genet Test Mol Biomarkers 17: 631-636, 2013.

79. Wang N, Li Y and Zhou J: Downregulation of ribonucleotide reductase subunits M2 induces apoptosis and G1 arrest of cervical cancer cells. Oncol Lett 15: 3719-3725, 2018

80. Mazumder Indra D, Mitra S, Singh RK, Dutta S, Roy A, Mondal RK, Basu PS, Roychoudhury S and Panda CK: Inactivation of CHEK1 and EI24 is associated with the development of invasive cervical carcinoma: Clinical and prognostic implications. Int J cancer 129: 1859-1871, 2011.

81. Zheng L, Li T, Zhang Y, Guo Y, Yao J, Dou L and Guo K Oncogene ATAD2 promotes cell proliferation, invasion and migration in cervical cancer. Oncol Rep 33: 2337-2344, 2015.

82. Cheng J, Lu X, Wang J, Zhang H, Duan P and Li C: Interactome analysis of gene expression profiles of cervical cancer reveals dysregulated mitotic gene clusters. Am J Transl Res 9: 3048-3059, 2017.
83. Mutch DG and Bloss JD: Gemcitabine in cervical cancer. Gynecol Oncol 90: S8-S15, 2003.

84. Roy S, Devleena, Maji T, Chaudhuri P, Lahiri D and Biswas J: Addition of gemcitabine to standard therapy in locally advanced cervical cancer: A randomized comparative study. Indian J Med Paediatr Oncol 32: 133-138, 2011.

85. Kalaghchi B, Abdi R, Amouzegar-Hashemi F, Esmati E and Alikhasi A: Concurrent chemoradiation with weekly paclitaxe and cisplatin for locally advanced cervical cancer. Asian Pac J Cancer Prev 17: 287-291, 2016.

86. Seidman AD: Gemcitabine as single-agent therapy in the management of advanced breast cancer. Oncology 15 (2 Suppl 3): S11-S14, 2001.

87. Wang E, Gulbis A, Hart JW and Nieto Y: The emerging role of gemcitabine in conditioning regimens for hematopoietic stem cell transplantation. Biol Blood Marrow Transplant 20: $1382-1389,2014$

88. Hu XC, Zhang J, Xu BH, Cai L, Ragaz J, Wang ZH, Wang BY, Teng YE, Tong ZS, Pan YY, et al: Cisplatin plus gemcitabine versus paclitaxel plus gemcitabine as first-line therapy for metastatic triple-negative breast cancer (CBCSG006): A randomised, open-label, multicentre, phase 3 trial. Lancet Oncol 16: 436-446, 2015.

89. Telli ML, Jensen KC, Vinayak S, Kurian AW, Lipson JA, Flaherty PJ, Timms K, Abkevich V, Schackmann EA, Wapnir IL, et al: Phase II study of gemcitabine, carboplatin, and iniparib as neoadjuvant therapy for triple-negative and BRCA1/2 mutation-associated breast cancer with assessment of a tumor-based measure of genomic instability: PrECOG 0105 J Clin Oncol 33: 1895-1901, 2015.

90. Park YH, Im SA, Kim SB, Sohn JH, Lee KS, Chae YS, Lee KH, Kim JH, Im YH, Kim JY, et al: Phase II, multicentre, randomised trial of eribulin plus gemcitabine versus paclitaxel plus gemcitabine as first-line chemotherapy in patients with HER2-negative metastatic breast cancer. Eur J Cancer 86: 385-393, 2017.

91. Niwińska A, Rudnicka $\mathrm{H}$ and Murawska M: Breast cancer leptomeningeal metastasis: The results of combined treatment and the comparison of methotrexate and liposomal cytarabine as intra-cerebrospinal fluid chemotherapy. Clin Breast Cancer 15: 66-72, 2015.

92. Laakmann E, Witzel I and Müller V: Efficacy of Liposomal Cytarabine in the treatment of leptomeningeal metastasis of breast cancer. Breast Care 12: 165-167, 2017.

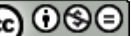

This work is licensed under a Creative Commons Attribution-NonCommercial-NoDerivatives 4.0 International (CC BY-NC-ND 4.0) License. 